\title{
Computing Deltas without derivatives
}

\author{
D. Baños • S. Duedahl • \\ T. Meyer-Brandis • F. Proske
}

Received: date / Accepted: date

\begin{abstract}
A well-known application of Malliavin calculus in Mathematical Finance is the probabilistic representation of option price sensitivities, the socalled Greeks, as expectation functionals that do not involve the derivative of the pay-off function. This allows for numerically tractable computation of the Greeks even for discontinuous pay-off functions. However, while the pay-off function is allowed to be irregular, the coefficients of the underlying diffusion are required to be smooth in the existing literature, which for example excludes already simple regime switching diffusion models. The aim of this article is to generalise this application of Malliavin calculus to Itô diffusions with irregular drift coefficients, whereat we here focus on the computation of the Delta, which is the option price sensitivity with respect to the initial value of the underlying. To this purpose we first show existence, Malliavin differentiability, and (Sobolev) differentiability in the initial condition of strong solutions of Itô diffusions with drift coefficients that can be decomposed into the sum of a bounded but merely measurable and a Lipschitz part. Furthermore, we

D. Baños

Department of Mathematics, University of Oslo, Moltke Moes vei 35, P.O. Box 1053 Blindern, 0316 Oslo, Norway.

E-mail: davidru@math.uio.no

S. Duedahl

Department of Mathematics, University of Oslo, Moltke Moes vei 35, P.O. Box 1053 Blindern, 0316 Oslo, Norway.

E-mail: sindred@math.uio.no

T. Meyer-Brandis

T. Meyer-Brandis: Department of Mathematics, LMU, Theresienstr. 39, D-80333 Munich, Germany.

E-mail: meyerbra@math.lmu.de

F. Proske

Department of Mathematics, University of Oslo, Moltke Moes vei 35, P.O. Box 1053 Blindern, 0316 Oslo, Norway.

E-mail: proske@math.uio.no
\end{abstract}


give explicit expressions for the corresponding Malliavin and Sobolev derivatives in terms of the local time of the diffusion, respectively. We then turn to the main objective of this article and analyse the existence and probabilistic representation of the corresponding Deltas for European and path-dependent options. We conclude with a small simulation study of several regime-switching examples.

Keywords Greeks · Delta · option sensitivities · Malliavin calculus · BismutElworthy-Li formula · irregular diffusion coefficients · strong solutions of stochastic differential equations $\cdot$ relative $L^{2}$-compactness

Mathematics Subject Classification (2010) MSC 60H10 - 60H07 .

$60 \mathrm{H} 30 \cdot 91 \mathrm{G} 60$

JEL (2019) C02 C63

\section{Introduction}

Throughout this paper, let $T>0$ be a given time horizon and $(\Omega, \mathcal{F}, P)$ a complete probability space equipped with a one-dimensional Brownian motion $\left\{B_{t}\right\}_{t \in[0, T]}$ and the filtration $\left\{\mathcal{F}_{t}\right\}_{t \in[0, T]}$ generated by $\left\{B_{t}\right\}_{t \in[0, T]}$ augmented by the $P$-null sets. Further, we will only deal with random variables that are Brownian functionals, i.e. we assume $\mathcal{F}:=\mathcal{F}_{T}$.

One of the most prominent applications of Malliavin calculus in financial mathematics concerns the derivation of numerically tractable expressions for the so-called Greeks, which are important sensitivities of option prices with respect to involved parameters. The first paper to address this application was 15, which has consecutively triggered an active research interest in this topic, see e.g. [14, 4], 11, 17], 21. See also [7, 11], 18] and references therein for a related approach based on functional Itô calculus. Suppose the risk-neutral dynamics of the underlying asset of a European option is driven by a stochastic differential equation (for short SDE) of the form

$$
d X_{t}^{x}=b\left(X_{t}^{x}\right) d t+\sigma\left(X_{t}^{x}\right) d B_{t}, \quad X_{0}^{x}=x \in \mathbb{R},
$$

where $b: \mathbb{R} \rightarrow \mathbb{R}$ and $\sigma: \mathbb{R} \rightarrow \mathbb{R}$ are some given drift and volatility coefficients, respectively. Let $\Phi: \mathbb{R} \rightarrow \mathbb{R}$ denote the pay-off function and the expectation $E\left[\Phi\left(X_{T}^{x}\right)\right]$ the risk-neutral price at time zero of the option with maturity $T>0$. For notational simplicity we assume the discounting rate to be zero. In this paper we will focus on the Delta

$$
\frac{\partial}{\partial x} E\left[\Phi\left(X_{T}^{x}\right)\right]
$$

which is a measure for the sensitivity of the option price with respect to changes of the initial value of the underlying asset. As is well known, the Delta has a particular role among the Greeks as it determines the hedge portfolio in many complete market models. If the drift $b(\cdot)$, the volatility $\sigma(\cdot)$, and the 
pay-off $\Phi(\cdot)$ are "sufficiently regular" to allow for differentiation under the expectation, the Delta can be computed in a straight-forward manner as

$$
E\left[\frac{\partial}{\partial x} \Phi\left(X_{T}^{x}\right)\right]=E\left[\Phi^{\prime}\left(X_{T}^{x}\right) Z_{T}\right]
$$

where the first variation process $Z_{t}:=\frac{\partial}{\partial x} X_{t}^{x}$ is given by

$$
Z_{t}=\exp \left\{\int_{0}^{t}\left[b^{\prime}\left(X_{s}^{x}\right)-\frac{1}{2}\left(\sigma^{\prime}\left(X_{s}^{x}\right)\right)^{2}\right] d s+\int_{0}^{t} \sigma^{\prime}\left(X_{s}^{x}\right) d B_{s}\right\}
$$

and where $\Phi^{\prime}, b^{\prime}, \sigma^{\prime}$ denote the derivatives of $\Phi, b, \sigma$, respectively. For example, requiring that $\Phi, b, \sigma$ are continuously differentiable with bounded derivatives would allow (2) to hold (we refer to 22] for conditions on $b$ and $\sigma$ that guarantee the existence of the first variation process), and the expectation in (2) could be approximated e.g. by Monte Carlo methods. In most realistic situations, though, straight-forward computations as in (2) are not possible. In this context, the following result obtained with the help of Malliavin calculus appears to be useful in particular for discontinuous pay-offs $\Phi$ as e.g. is the case for digital options.

Theorem 1.1 (Proposition 3.2 in [15]). Let $b(\cdot)$ and $\sigma(\cdot)$ be continuously differentiable with bounded Lipschitz derivatives, $\sigma(\cdot)>\epsilon>0$, and $\Phi\left(X_{T}^{x}\right)$ be square integrable. Then the Delta exists and is given by

$$
\frac{\partial}{\partial x} E\left[\Phi\left(X_{T}^{x}\right)\right]=E\left[\Phi\left(X_{T}^{x}\right) \int_{0}^{T} a(t) \sigma^{-1}\left(X_{t}^{x}\right) Z_{t} d B_{t}\right],
$$

where $Z_{t}$ is the first variation process given in (3) and $a(t)$ is any square integrable deterministic function such that

$$
\int_{0}^{T} a(s) d s=1
$$

While for notational simplicity we present the above result for one - dimensional $X^{x}$ we remark that in [15] the extension to the multi-dimensional setting is considered. We remark that the probabilistic representation (4) of the space derivative of the solution to the associated Kolmogorov equation is also referred to as Bismuth-Elworthy-Li type formula in the literature due to [13, 6]. The strength of (4) is that the Delta is expressed again as an expectation of the pay-off multiplied by the so-called Malliavin weight $\int_{0}^{T} a(t) \sigma^{-1}\left(X_{t}^{x}\right) Z_{t} d B_{t}$. Computing the Delta by Monte-Carlo via this reformulation then guarantees a convergence rate that is independent of the regularity of the pay-off function $\Phi$ and the dimensionality. Note that the Malliavin weight is independent of the option pay-off, and thus the same weight can be employed in the computations of the Deltas of different options. Also, in [14] and [3] the question of how to optimally choose the function $a(t)$ with respect to computational efficiency is considered. 
While the representation (4) succeeds to handle irregular pay-offs by getting rid of the derivative of $\Phi$, the regularity assumptions on the coefficients $b$ and $\sigma$ driving the dynamics of the underlying diffusion are rather strong. Consider for example an extended Black and Scholes model where the stock pays a dividend yield that switches to a higher level when the stock value passes a certain threshold. Then, again with the risk-free rate equal to zero for simplicity, the logarithm of the stock price is modelled by the following dynamics under the risk-neutral measure:

$$
d X_{t}^{x}=b\left(X_{t}^{x}\right) d t+\sigma d B_{t}, \quad X_{0}^{x}=x \in \mathbb{R},
$$

where $\sigma>0$ is constant and the drift coefficient $b: \mathbb{R} \rightarrow \mathbb{R}$ is given by

$$
b(x):=-\lambda_{1} \mathbf{1}_{(-\infty, R)}(x)-\lambda_{2} \mathbf{1}_{[R, \infty)}(x)-\frac{\sigma^{2}}{2},
$$

for dividend yields $\lambda_{1}, \lambda_{2} \in \mathbb{R}_{+}$and a given threshold $R \in \mathbb{R}$. Also, in [9] a (more complex) irregular drift $b$ is interpreted as state-dependent fees deducted by the insurer in the evolution of variable annuities instead of dividend yield. Already, this simple regime-switching model is not covered by the result in Theorem 1.1 since the drift coefficient is not continuously differentiable.

Or allow for state-dependent regime-switching of the mean reversion rate in an extended Ornstein-Uhlenbeck process:

$$
d X_{t}^{x}=b\left(X_{t}^{x}\right) d t+\sigma d B_{t}, \quad X_{0}^{x}=x \in \mathbb{R},
$$

where $\sigma>0$ is constant and the drift coefficient $b: \mathbb{R} \rightarrow \mathbb{R}$ is given by

$$
b(x):=-\lambda_{1} x \mathbf{1}_{(-\infty, R)}(x)-\lambda_{2} x \mathbf{1}_{[R, \infty)}(x)
$$

for mean reversion rates $\lambda_{1}, \lambda_{2} \in \mathbb{R}_{+}$and a given threshold $R \in \mathbb{R}$ (here the mean reversion level is set equal to zero). This type of model captures well, for instance, the evolution of (de-seasonalized) electricity spot prices, which switches between so-called spike regimes on high price levels with very fast mean reversion and base regimes on normal price levels with moderate speed of mean reversion, see e.g. [5], 20], 29] and references therein. Alternatively, an extended Ornstein-Uhlenbeck process with state-dependent regime-switching of the mean reversion level (low and high interest rate environments) is an interesting modification of the Vašíček short rate model. Note that in that case the Delta is rather a generalised Rho, i.e. a sensitivity measure with respect to the short end of the yield curve. We observe that also these two extended Ornstein-Uhlenbeck processes are not covered by the result in Theorem 1.1.

Motivated by these examples, this paper aims at deriving an analogous result to Theorem 1.1 when the underlying is driven by an SDE with irregular drift coefficient. More precisely, we will consider SDE's

$$
d X_{t}^{x}=b\left(t, X_{t}^{x}\right) d t+d B_{t}, 0 \leq t \leq T, X_{0}^{x}=x \in \mathbb{R},
$$


where we allow for time-inhomogeneous drift coefficients $b:[0, T] \times \mathbb{R} \rightarrow \mathbb{R}$ in the form

$$
b(t, x)=\tilde{b}(t, x)+\hat{b}(t, x), \quad(t, x) \in[0, T] \times \mathbb{R},
$$

for $\tilde{b}$ merely bounded and measurable, and $\hat{b}$ Lipschitz continuous and at most of linear growth in $x$ uniformly in $t$, i.e. there exists a constant $C>0$ such that

$$
\begin{aligned}
|\hat{b}(t, x)-\hat{b}(t, y)| & \leq C|x-y| \\
|\hat{b}(t, x)| & \leq C(1+|x|)
\end{aligned}
$$

for $x, y \in \mathbb{R}$ and $t \in[0, T]$. Adding the Lipschitz component $\hat{b}(t, x)$ in $(6)$ is motivated by the fact that many drift coefficients interesting for financial applications are of linear growths. At present we are not able to show our results for general measurable drift coefficients of linear growths, but only for those where the irregular behaviour remains in a bounded spectrum. However, from an application point of view this class is very rich already, and in particular it contains the regime switching examples from above. We remark that while in (5) we consider a constant volatility coefficient $\sigma(t, x):=1$, we will see at the end of Section 3 (Theorem 3.8) that our results apply to many SDE's with more general volatility coefficients which can be reduced to SDE's of type (5) (which for example is possible for volatility coefficients as in Theorem 1.1).

In order to be able to apply Malliavin calculus to the underlying diffusion, the first thing we need to ensure is that the solution of SDE (5) is a Brownian functional, i.e. we are interested in the existence of strong solutions of (5).

Definition 1.2. A strong solution of SDE (5) is a continuous $\left\{X_{t}^{x}\right\}_{t \in[0, T]}$, $\left\{\mathcal{F}_{t}\right\}_{t \in[0, T]}$-adapted process that solves equation (5).

Remark 1.3. Note that the usual definition of a strong solution requires the existence of a Brownian-adapted solution of (5) on any given stochastic basis. However, an $\left\{\mathcal{F}_{t}\right\}_{t \in[0, T]}$-adapted solution $\left\{X_{t}^{x}\right\}_{t \in[0, T]}$ on the stochastic basis $(\Omega, \mathcal{F}, P, B)$ can be written in the form $X_{t}^{x}=F_{t}(B$. ) for some family of functionals $F_{t}, t \in[0, T]$, (see e.g. [27] for an explicit form of $F_{t}$ ). Then for any other stochastic basis $(\hat{\Omega}, \hat{\mathcal{F}}, \hat{P}, \hat{B})$ one gets that $X_{t}^{x}:=F_{t}(\hat{B}),. t \in[0, T]$, is a $\hat{B}$-adapted solution to SDE $(5)$. So once there is a Brownian-adapted solution of (5) on one given stochastic basis, it follows that there indeed exists a strong solution in the usual sense. This justifies our definition of a strong solution above.

To pursue our objectives we proceed as follows in the remaining parts of the paper. In Section 2 we recall some fundamental concepts from Malliavin calculus and local time calculus which compose central mathematical tools in the following analysis.

We then analyse in Section 3 the existence and Malliavin differentiability of a unique strong solution of SDE's with irregular drift coefficients as in (5) (Theorem 3.1). It is well known that the SDE is Malliavin differentiable as soon as the coefficients are Lipschitz continuous (see e.g. [31]); for 
merely bounded and measurable drift coefficients Malliavin differentiability was shown only recently in [28, (see also [26]). Here, we extend ideas introduced for bounded coefficients in [28] to drift coefficients of type (6). Unlike in most of the existing literature on strong solutions of SDE's with irregular coefficients our approach does not rely on a pathwise uniqueness argument (Yamada-Watanabe theorem). Instead, we employ a compactness criterium based on Malliavin calculus together with local time calculus to directly construct a strong solution which in addition is Malliavin differentiable. Also, we are able to give an explicit expression for the Malliavin derivative of the strong solution of (5) in terms of the integral of $b$ (and not the derivative of $b$ ) with respect to local time of the strong solution (Proposition 3.2). We mention that while existence and Malliavin differentiability of strong solutions could be extended to analogue multi-dimensional SDE's as in [26, the explicit expression of the Malliavin derivative considered in this paper is in general only possible for one-dimensional SDE's. Moreover, in this paper we replace arguments that are based on White Noise analysis in 28] and [26] by alternative proofs which might make the text more accessible for readers who are unfamiliar with concepts from White Noise analysis.

Next, we need to analyse the regularity of the dependence of the strong solution in its initial condition and to introduce the analogue of the first variation process $(3)$ in case of irregular drift coefficients. Using the close connection between the Malliavin derivative and the first variation process, we find that the strong solution is Sobolev differentiable in its initial condition (Theorem 3.4). Again, we give an explicit expression for the corresponding (Sobolev) first variation process which does not include the derivative of $b$ (Proposition 3.5).

In Section 4 we develop our main result (Theorem 4.2) which extends Theorem 1.1 to SDE's with irregular drift coefficients. To this end, we need to show in the first place that the Delta exists, i.e. that $E\left[\Phi\left(X_{T}^{x}\right)\right]$ is continuously differentiable in $x$. At this point the explicit expressions for the Malliavin derivative and the first variation process are essential. In the final representation of the Delta we then have gotten rid of both the derivative of the pay-off $\Phi$ and the derivative of the drift coefficient $b$ in the first variation process, whence the title "Computing Deltas without Derivatives" of the paper. We then proceed by extending the result to path-dependent options, which is possible for those pay-offs that allow for "a time window to employ integration-by-parts".

In Section 5 we consider some examples and compute the Deltas in the concrete regime-switching models mentioned above. We do a small simulation study and compare the performance to a finite difference approximation of the Delta in the same spirit as in [15].

We conclude the paper by an appendix with some technical proofs from Section 3 which have been deferred to the end of the paper for better readability.

Notations: We summarise some of the most frequently used notations:

- We denote by $C(\mathbb{R})$, respectively $C^{1}(\mathbb{R})$, the space of continuous functions $f: \mathbb{R} \rightarrow \mathbb{R}$, respectively continuously differentiable functions $f: \mathbb{R} \rightarrow \mathbb{R}$. 
$-C_{0}^{\infty}([0, T] \times \mathbb{R})$, respectively $C_{0}^{\infty}(\mathbb{R})$, denotes the space of infinitely many times differentiable functions on $[0, T] \times \mathbb{R}$, respectively $\mathbb{R}$, with compact support.

- For a measurable space $(S, \mathcal{G})$ equipped with a measure $\mu$, we denote by $L^{p}(S, \mathcal{G})$ or $L^{p}(S)$ the Banach space of (equivalence classes of) functions on $S$ integrable to some power $p, p \geq 1$.

$-L_{\text {loc }}^{p}(\mathbb{R})$ denotes the space of locally Lebesgue integrable functions to some power $p, p \geq 1$, i.e. $\int_{U}|f(x)|^{p} d x<\infty$ for every open bounded subset $U \subset \mathbb{R}$.

- $W_{l o c}^{1, p}(\mathbb{R})$ denotes the subspace of $L_{l o c}^{p}(\mathbb{R})$ of weakly (Sobolev) differentiable functions such that the weak derivative $f^{\prime}$ belongs to $L_{l o c}^{p}(\mathbb{R}), p \geq 1$.

- For a progressive process $Y$. we denote the Doléans-Dade exponential of the corresponding Brownian integral (if well-defined) by

$$
\begin{aligned}
& \mathcal{E}\left(\int_{0}^{t} b\left(u, Y_{u}\right) d B_{u}\right) \\
& \quad:=\exp \left(\int_{0}^{t} b\left(u, Y_{u}\right) d B_{u}-\frac{1}{2} \int_{0}^{t} b^{2}\left(u, Y_{u}\right) d u\right), \quad t \in[0, T] .
\end{aligned}
$$

To shorten the writing we will sometimes simply use the notation

$$
\mathcal{E}(b)_{t}:=\mathcal{E}\left(\int_{0}^{t} b\left(u, Y_{u}\right) d B_{u}\right), \quad t \in[0, T] .
$$

- For $Z \in L^{2}\left(\Omega, \mathcal{F}_{T}\right)$ we denote the Wiener-transform of $Z$ in $f \in L^{2}([0, T])$ by

$$
\mathcal{W}(Z)(f):=E\left[Z \mathcal{E}\left(\int_{0}^{T} f(s) d B_{s}\right)\right] .
$$

- Throughout the paper, we will denote $B_{t}^{x}:=x+B_{t}, t \in[0, T]$ and simply $B_{t}:=B_{t}^{0}$ when $x=0$ for the standard Brownian motion $B$.

- We will use the symbol $\lesssim$ to denote less or equal than up to a positive real constant $C>0$ not depending on the parameters of interest, i.e. if we have two mathematical expressions $E_{1}(\theta), E_{2}(\theta)$ depending on some parameter of interest $\theta$ then $E_{1}(\theta) \lesssim E_{2}(\theta)$ if, and only if, there is a positive real number $C>0$ independent of $\theta$ such that $E_{1}(\theta) \leq C E_{2}(\theta)$.

\section{Framework}

Our main results centrally rely on tools from Malliavin calculus as well as integration with respect to local time both in time and space. We here provide a concise introduction to the main concepts in these two areas that will be employed in the following sections. For deeper information on Malliavin calculus the reader is referred to i.e. [31, 24, 25, 10]. As for theory on local time integration for Brownian motion we refer to i.e. [12, 32]. 
2.1 Malliavin calculus

Denote by $\mathbb{D}^{1,2} \subset L^{2}(\Omega)$ the space of Malliavin differentiable random variables and further by $D_{t} F \in L^{2}(\Omega \times[0, T])$ the Malliavin derivative of $F \in \mathbb{D}^{1,2}$. In the derivation of the probabilistic representation for the Delta, the following chain rule and duality formula for the Malliavin derivative will be essential:

Lemma 2.1. Let $\varphi: \mathbb{R}^{m} \rightarrow \mathbb{R}$ be continuously differentiable with bounded partial derivatives. Further, suppose that $F=\left(F_{1}, \ldots, F_{m}\right)$ is a random vector whose components are in $\mathbb{D}^{1,2}$. Then $\varphi(F) \in \mathbb{D}^{1,2}$ and

$$
D_{t} \varphi(F)=\sum_{i=1}^{m} \partial_{i} \varphi(F) D_{t} F_{i}, \quad P-a . s ., \quad t \in[0, T] .
$$

Theorem 2.2 (Duality formula). Let $u(t)$ be a square-integrable, adapted process and $F \in \mathbb{D}^{1,2}$. Then

$$
E\left[F \int_{0}^{T} u_{t} d B_{t}\right]=E\left[\int_{0}^{T} u_{t} D_{t} F d t\right] .
$$

The next result, which is due to [8] and central in proving existence of strong solutions in the following, provides a compactness criterion for subsets of $L^{2}(\Omega)$ based on Malliavin calculus.

Proposition 2.3. Let $F_{n} \in \mathbb{D}^{1,2}, n=1,2 \ldots$, be a given sequence of Malliavin differentiable random variables. Assume that there exist constants $\alpha>0$ and $C>0$ such that

$$
\begin{gathered}
\sup _{n} E\left[\left|F_{n}\right|^{2}\right] \leq C, \\
\sup _{n} E\left[\left|D_{t} F_{n}-D_{t^{\prime}} F_{n}\right|^{2}\right] \leq C\left|t-t^{\prime}\right|^{\alpha}
\end{gathered}
$$

for $0 \leq t^{\prime} \leq t \leq T$, and

$$
\sup _{n} \sup _{0 \leq t \leq T} E\left[\left|D_{t} F_{n}\right|^{2}\right] \leq C
$$

Then the sequence $F_{n}, n=1,2 \ldots$, is relatively compact in $L^{2}(\Omega)$.

We conclude this review on Malliavin calculus by stating a relation between the Malliavin derivative and the first variation process of the solution of an SDE with smooth coefficients that is essential in the derivation of Theorem 1.1 We give the result for the case when the volatility coefficient is equal to 1 , but the analogue result is valid for more general smooth volatility coefficients. Assume the drift coefficient $b(t, x)$ in the SDE (5) fulfils the Lipschitz and linear growth conditions (7)-(8). Then it is well-known that there exists a unique strong solution $X_{t}^{x}, t \in[0, T]$, to equation (5) that is Malliavin differentiable, and that for all $0 \leq s \leq t \leq T$ the Malliavin derivative $D_{s} X_{t}^{x}$ fulfils, see e.g. [31, Theorem 2.2.1]

$$
D_{s} X_{t}^{x}=1+\int_{s}^{t} b^{\prime}\left(u, X_{u}^{x}\right) D_{s} X_{u}^{x} d u,
$$


where $b^{\prime}$ denotes the (weak) derivative of $b$ with respect to $x$.

Further, under these assumptions the strong solution is also differentiable in its initial condition, and the first variation process $\frac{\partial}{\partial x} X_{t}^{x}, t \in[0, T]$ fulfills (see e.g. 22] for differentiable coefficients and [2] for an extension to Lipschitz coefficients)

$$
\frac{\partial}{\partial x} X_{t}^{x}=1+\int_{0}^{t} b^{\prime}\left(u, X_{u}^{x}\right) \frac{\partial}{\partial x} X_{u}^{x} d u .
$$

Solving equations 10 and 11 thus yields the following proposition.

Proposition 2.4. Let $X_{t}^{x}, t \in[0, T]$, be the unique strong solution to equation (5) when $b(t, x)$ fulfills the Lipschitz and linear growth condition (7)-(8). Then $\bar{X}_{t}^{x}$ is Malliavin differentiable and differentiable in its initial condition for all $t \in[0, T]$, and for all $s \leq t \leq T$ we have

$D_{s} X_{t}^{x}=\exp \left\{\int_{s}^{t} b^{\prime}\left(u, X_{u}^{x}\right) d u\right\} \quad$ and $\quad \frac{\partial}{\partial x} X_{t}^{x}=\exp \left\{\int_{0}^{t} b^{\prime}\left(u, X_{u}^{x}\right) d u\right\}$.

As a consequence,

$$
\frac{\partial}{\partial x} X_{t}^{x}=D_{s} X_{t}^{x} \frac{\partial}{\partial x} X_{s}^{x}
$$

2.2 Integration with respect to local-time

Let $X^{x}$ be a given (strong) solution to SDE (5). In the sequel we need the concept of stochastic integration over the plane with respect to the local time $L^{X^{x}}(t, y)$ of $X^{x}$. For Brownian motion, the local time integration theory in time and space has been introduced in 12 . We extend this local time integration theory to more general diffusions of type (5) by resorting to the Brownian setting under an equivalent measure where $X^{x}$ is a Brownian motion. To this end, we first notice the following two facts that are extensively used throughout the paper:

Remark 2.5. Since the drift coefficient $b$ is of at most linear growth Beneš' result, see e.g. [19, Corollary 5.16], implies that the Radon-Nikodym density

$$
\frac{d Q}{d P}=\mathcal{E}\left(-\int_{0}^{T} b\left(s, X_{s}^{x}\right) d B_{s}\right)
$$

defines a probability measure $Q$ equivalent to $P$. Under $Q$, the solution $X^{x}$ is Brownian motion starting in $x$ by Girsanov's theorem.

Lemma 2.6. Let $b:[0, T] \times \mathbb{R} \rightarrow \mathbb{R}$ be a function of at most linear growth, i.e. $|b(t, x)| \leq C(1+|x|)$ for some $C>0$, all $x \in \mathbb{R}$ and $t \in[0, T]$. Then for any compact subset $K \subset \mathbb{R}$ there exists an $\varepsilon>0$ such that

$$
\sup _{x \in K} E\left[\mathcal{E}\left(\int_{0}^{T} b\left(u, B_{u}^{x}\right) d B_{u}\right)^{1+\varepsilon}\right]<\infty .
$$


Proof. Indeed, write

$$
\begin{aligned}
E\left[\mathcal{E}\left(\int_{0}^{T} b\left(u, B_{u}^{x}\right) d B_{u}\right)^{1+\varepsilon}\right] \\
=E\left[\exp \left\{\int_{0}^{T}(1+\varepsilon) b\left(u, B_{u}^{x}\right) d B_{u}-\frac{1}{2} \int_{0}^{T}(1+\varepsilon) b^{2}\left(u, B_{u}^{x}\right) d u\right\}\right] \\
=E\left[\operatorname { e x p } \left\{\int_{0}^{T}(1+\varepsilon) b\left(u, B_{u}^{x}\right) d B_{u}-\frac{1}{2} \int_{0}^{T}(1+\varepsilon)^{2} b^{2}\left(u, B_{u}^{x}\right) d u\right.\right. \\
\left.\left.+\frac{1}{2} \int_{0}^{T} \varepsilon(1+\varepsilon) b^{2}\left(u, B_{u}^{x}\right) d u\right\}\right] \\
=E\left[\exp \left\{\frac{1}{2} \int_{0}^{T} \varepsilon(1+\varepsilon) b^{2}\left(u, X_{u}^{\varepsilon, x}\right) d u\right\}\right],
\end{aligned}
$$

where in the last step by Girsanov's theorem $X^{\varepsilon, x}$ denotes a weak solution of the SDE

$$
\left\{\begin{array}{l}
d X_{t}^{\varepsilon, x}=(1+\varepsilon) b\left(t, X_{t}^{\varepsilon, x}\right) d t+d B_{t}, \quad t \in[0, T] \\
X_{0}^{\varepsilon, x}=x .
\end{array}\right.
$$

Observe that, since $b$ has at most linear growth, we have

$$
\left|X_{t}^{\varepsilon, x}\right| \leq|x|+C(1+\varepsilon) \int_{0}^{t}\left(1+\left|X_{u}^{\varepsilon, x}\right|\right) d u+\left|B_{t}\right|
$$

for every $t \in[0, T]$. Then Grönwall's inequality gives

$$
\left|X_{t}^{\varepsilon, x}\right| \leq\left(|x|+C(1+\varepsilon) T+\left|B_{t}\right|\right) e^{C(1+\varepsilon) T},
$$

Due to the sublinearity of $b$ and (14) we can find a constant $C_{\varepsilon, T}$ depending only on $\varepsilon, T$ such that $\lim _{\varepsilon \succ 0} C_{\varepsilon, T}<\infty$ and

$$
\left|b\left(u, X_{u}^{\varepsilon, x}\right)\right| \leq C_{\varepsilon, T}\left(1+|x|+\left|B_{t}\right|\right) .
$$

Then

$$
\begin{aligned}
& E\left[\exp \left\{\varepsilon(1+\varepsilon) \int_{0}^{T} b^{2}\left(u, X_{u}^{\varepsilon, x}\right) d u\right\}\right] \\
& \quad \leq E\left[\exp \left\{\varepsilon(1+\varepsilon) C_{\varepsilon, T}^{2} \int_{0}^{T}\left(1+|x|+\left|B_{u}\right|\right)^{2} d u\right\}\right] \\
& \leq e^{\tilde{C}_{\varepsilon, T} T(1+|x|)^{2}} E\left[\exp \left\{2 \tilde{C}_{\varepsilon, T}(1+|x|) \int_{0}^{T}\left|B_{u}\right| d u+\tilde{C}_{\varepsilon, T} \int_{0}^{T}\left|B_{u}\right|^{2} d u\right\}\right],
\end{aligned}
$$


where $\tilde{C}_{\varepsilon, T}:=\varepsilon(1+\varepsilon) C_{\varepsilon, T}^{2}>0$ is a constant such that $\lim _{\varepsilon \backslash 0} \tilde{C}_{\varepsilon, T}=0$. Clearly, from the above expression we can see that for every compact set $K \subset \mathbb{R}$ we can choose $\varepsilon>0$ small enough such that

$$
\sup _{x \in K} E\left[\exp \left\{\varepsilon(1+\varepsilon) \int_{0}^{T} b^{2}\left(u, X_{u}^{\varepsilon, x}\right) d u\right\}\right]<\infty .
$$

We define the feasible integrands for the local time-space integral w.r.t. $L^{X^{x}}(t, y)$ by the Banach space $\left(\mathcal{H}^{x},\|\cdot\|\right)$ of functions $f:[0, T] \times \mathbb{R} \longrightarrow \mathbb{R}$ with norm

$$
\begin{aligned}
\|f\|_{x}= & 2\left(\int_{0}^{T} \int_{\mathbb{R}} f^{2}(s, y) \frac{1}{\sqrt{2 \pi s}} \exp \left(-\frac{|y-x|^{2}}{2 s}\right) d y d s\right)^{1 / 2} \\
& +\int_{0}^{T} \int_{\mathbb{R}}|y-x||f(s, y)| \frac{1}{s \sqrt{2 \pi s}} \exp \left(-\frac{|y-x|^{2}}{2 s}\right) d y d s .
\end{aligned}
$$

We remark that this space of integrands is the same as the one introduced in 12 for Brownian motion, except that we have in a straight forward manner generalised the space in [12] to the situation when the Brownian motion has arbitrary initial value $x$.

We denote by $f_{\Delta}:[0, T] \times \mathbb{R} \longrightarrow \mathbb{R}$ a simple function in the form

$$
f_{\Delta}(s, y)=\sum_{1 \leq i \leq n-1,1 \leq j \leq m-1} f_{i j} \mathbf{1}_{\left(y_{i}, y_{i+1}\right]}(y) \mathbf{1}_{\left(s_{j}, s_{j+1}\right]}(s),
$$

where $\left(s_{j}\right)_{1 \leq j \leq m}$ is a partition of $[0, T]$ and $\left(y_{i}\right)_{1 \leq i \leq n}$ and $\left(f_{i j}\right)_{1 \leq i \leq n, 1 \leq j \leq m}$ are finite sequences of real numbers. It is readily checked that the space of simple functions is dense in $\left(\mathcal{H}^{x},\|\cdot\|\right)$. The local time-space integral of an simple function $f_{\Delta}$ with respect to $L^{X^{x}}(d t, d y)$ is then defined by

$$
\begin{aligned}
& \int_{0}^{T} \int_{\mathbb{R}} f_{\Delta}(s, y) L^{X^{x}}(d s, d y):= \\
& \sum_{\substack{1 \leq i \leq n-1 \\
1 \leq j \leq m-1}} f_{i j}\left(L^{X^{x}}\left(s_{j+1}, y_{i+1}\right)-L^{X^{x}}\left(s_{j}, y_{i+1}\right)-L^{X^{x}}\left(s_{j+1}, y_{i}\right)+L^{X^{x}}\left(s_{j}, y_{i}\right)\right) .
\end{aligned}
$$

Lemma 2.7. For $f \in \mathcal{H}^{x}$ let $f_{n}, n \geq 1$, be a sequence of simple functions converging to $f$ in $\mathcal{H}^{x}$. Then $\int_{0}^{T} \int_{\mathbb{R}} f_{n}(s, y) L^{X^{x}}(d s, d y), n \geq 1$, converges in probability. Further, for any other approximating sequence of simple functions the limit remains the same.

Proof. Define $F_{n}^{X^{x}}:=\int_{0}^{T} \int_{\mathbb{R}} f_{n}(s, x) L^{X^{x}}(d s, d x)$. Now consider the equivalent measure $Q$ from Remark 2.5 under which $X^{x}$ is Brownian motion. Define $F^{X^{x}}:=\int_{0}^{T} \int_{\mathbb{R}} f(s, x) L^{X^{x}}(d s, d x)$ to be the time-space integral of $f$ with respect to the local time of Brownian motion $X^{x}$ under $Q$, which exists as an 
$L^{1}(Q)$-limit of $F_{n}^{X^{x}}, n \geq 1$ by the Brownian local time integration theory introduced in [12] (since $f_{n}, n \geq 1$ converges to $f$ in $\mathcal{H}^{x}$ ). We show that $F_{n}^{X^{x}}, n \geq 1$ converges in probability to $F^{X^{x}}$ under $P$. Indeed,

$$
\begin{aligned}
& E\left[1 \wedge\left|F^{X^{x}}-F_{n}^{X^{x}}\right|\right]=E\left[\left(1 \wedge\left|F^{B^{x}}-F_{n}^{B^{x}}\right|\right) \mathcal{E}\left(\int_{0}^{T} b\left(s, B_{s}^{x}\right) d B_{s}\right)\right] \\
& \quad \leq E\left[\mathcal{E}\left(\int_{0}^{T} b\left(s, B_{s}^{x}\right) d B_{s}\right)^{1+\varepsilon}\right]^{1 /(1+\varepsilon)} E\left[\left(1 \wedge\left|F^{B^{x}}-F_{n}^{B^{x}}\right|\right)^{\frac{1+\varepsilon}{\varepsilon}}\right]^{\frac{\varepsilon}{1+\varepsilon}} \\
& \quad \leq C_{\varepsilon} E\left[\left(1 \wedge\left|F^{B^{x}}-F_{n}^{B^{x}}\right|\right)\right] \stackrel{\frac{\varepsilon}{1+\varepsilon}}{\stackrel{n \rightarrow \infty}{\longrightarrow} 0},
\end{aligned}
$$

where, in analogy to the notation $F^{X^{x}}$ and $F_{n}^{X^{x}}$ above, the notation $F^{B^{x}}$ and $F_{n}^{B^{x}}$ refers to the corresponding integrals with respect to local time of Brownian motion $B^{x}$ under $P$, and where in the first equality we have used that $\left(F^{B^{x}}, F_{n}^{B^{x}}\right)$ has the same law under $P$ as $\left(F^{X^{x}}, F_{n}^{X^{x}}\right)$ under $Q$. The inequalities follow by Lemma 2.6 for some $\varepsilon>0$ suitably small. Further, by 12] we know that $F_{n}^{B^{x}}, n \geq 1$ converges to $F^{B^{x}}$ in $L^{1}(P)$, which implies the convergence in 15. Hence $F_{n}^{X^{x}}, n \geq 1$ converge to $F^{X^{x}}$ in the Ky-Fan metric $d(X, Y)=E[1 \wedge|X-Y|], X, Y \in L^{0}(\Omega)$, which characterises convergence in probability. Finally, again by [12], $F^{X^{x}}$ is independent of the approximating sequence $f_{n}, n \geq 1$.

Definition 2.8. For $f \in \mathcal{H}^{x}$, the limit in Lemma 2.7 will be denoted by $\int_{0}^{T} \int_{\mathbb{R}} f(s, y) L^{X^{x}}(d s, d y)$ and is called the time-space integral of $f$ with respect to $L^{X^{x}}(d t, d x)$. Further, for any $t \in[0, T]$ we define

$$
\int_{0}^{t} \int_{\mathbb{R}} f(s, y) L^{X^{x}}(d s, d y):=\int_{0}^{T} \int_{\mathbb{R}} f(s, y) I_{[0, t]}(s) L^{X^{x}}(d s, d y) .
$$

Remark 2.9. We notice that the drift coefficient $b(t, x)$ in (6), which is of linear growth in $x$ uniformly in $t$, is in $\mathcal{H}^{x}$, and thus the local time integral of $b(t, x)$ with respect to $L^{X^{x}}(d t, d y)$ exists for any $x \in \mathbb{R}$.

If $X^{x}$ is a Brownian motion $B^{x}$ we have the following decomposition due to [12] that we employ in the construction of strong solutions, and that also constitutes the foundation in the construction of the local time integral in [12.

Theorem 2.10. Let $f \in \mathcal{H}^{0}$. Then

$$
\begin{aligned}
\int_{0}^{t} & \int_{\mathbb{R}} f(s, y) L^{B^{x}}(d s, d y)= \\
& =\int_{0}^{t} f\left(s, B_{s}^{x}\right) d B_{s}+\int_{T-t}^{T} f\left(T-s, \widehat{B}_{s}^{x}\right) d W_{s}-\int_{T-t}^{T} f\left(T-s, \widehat{B}_{s}^{x}\right) \frac{\widehat{B}_{s}}{T-s} d s,
\end{aligned}
$$

where $\widehat{B}_{t}:=B_{T-t}, 0 \leq t \leq T$ is the time-reversed Brownian motion, and $W_{t}:=\widehat{B}_{t}-B_{T}+\int_{0}^{t} \frac{\widehat{B}_{s}}{T-s} d s$ is a Brownian motion with respect to the filtration of $\widehat{B}$. 
We conclude this subsection by stating three further identities for the local time integral for a general diffusion $X^{x}$ which will be useful later on.

Lemma 2.11. Let $f \in \mathcal{H}^{x}$ be Lipschitz continuous in $x$. Then for all $t \in[0, T]$

$$
-\int_{0}^{t} \int_{\mathbb{R}} f(s, y) L^{X^{x}}(d s, d y)=\int_{0}^{t} f^{\prime}\left(s, X_{s}^{x}\right) d s .
$$

where $f^{\prime}$ denotes the (weak) derivative of $f(t, y)$ with respect to $y$.

If $f \in \mathcal{H}^{x}$ is time homogeneous (i.e. $f(t, y)=f(y)$ only depends on the space variable) and locally square integrable, then for any $t \in[0, T]$

$$
\int_{0}^{t} \int_{\mathbb{R}} f(s, x) L^{X^{x}}(d s, d x)=-\left[f\left(\cdot, X^{x}\right), X^{x}\right]_{t}
$$

and

$$
-\int_{0}^{t} \int_{\mathbb{R}} f(s, y) L^{X^{x}}(d s, d y)=2 F\left(X_{t}^{x}\right)-2 F(x)-2 \int_{0}^{t} f\left(X_{s}^{x}\right) d X_{s}^{x},
$$

where $F$ is a primitive function of $f$ and $\left[\tilde{b}\left(\cdot, X^{x}\right), X^{x}\right]_{t}$ is the generalised covariation process

$\left[f\left(\cdot, X_{.}^{x}\right), X_{.}^{x}\right]_{t}:=P-\lim _{m \rightarrow \infty} \sum_{k=1}^{m}\left(f\left(t_{k}^{m}, X_{t_{k}^{m}}^{x}\right)-f\left(t_{k-1}^{m}, X_{t_{k-1}^{m}}^{x}\right)\right)\left(X_{t_{k}}^{x}-X_{t_{k-1}}^{x}\right)$,

where for every $m$ we denote by $\left\{t_{k}^{m}\right\}_{k=1}^{m}$ a partition of the interval $[0, t]$ such that $\lim _{m} \sup _{k=1, \ldots, m}\left|t_{k}^{m}-t_{k-1}^{m}\right|=0$. Note that 19 can be considered as a generalised Itô formula.

Proof. If $X^{x}=x+B$, then identities 17$)-(19)$ are given in 12] (where these identities are given for the case $x=0$, but one can easily extend them to the case of the Brownian motion starting at an arbitrary $x \in \mathbb{R}$ ). For general $X^{x}$, we consider the identities under the equivalent measure $Q$ from Remark 2.5 Then, by the construction of the local time integral outlined in Lemma 2.7. the integrals in the identities are the ones with respect to Brownian motion $X^{x}$, for which we know the identities hold by [12].

\section{Existence, Malliavin, and Sobolev differentiability of strong solutions}

In this section we prepare the necessary theoretical grounds to develop the probabilistic representation of Deltas. Being notationally and technically rather heavy, the proofs of this section are deferred to Appendix A for an improved flow and readability of the paper. We first study the existence and Malliavin differentiability of a unique strong solution of SDE (5) before we turn to the differentiability of the strong solution in its initial condition and the corresponding first variation process. We state the first main result of this section: 
Theorem 3.1. Suppose that the drift coefficient $b:[0, T] \times \mathbb{R} \rightarrow \mathbb{R}$ is in the form (6). Then there exists a unique strong solution $\left\{X_{t}^{x}\right\}_{t \in[0, T]}$ to SDE (5). In addition, $X_{t}^{x}$ is Malliavin differentiable for every $t \in[0, T]$.

The proof of Theorem 3.1 employs several auxiliary results presented in Appendix A. The main steps are:

1. First, we construct a weak solution $X^{x}$ to (5) by means of Girsanov's theorem, that is we introduce a probability space $(\Omega, \mathcal{F}, P)$ that carries some Brownian motion $B$ and a continuous process $X^{x}$ such that $(5)$ is fulfilled. However, a priori $X^{x}$ is not adapted to the filtration $\left\{\mathcal{F}_{t}\right\}_{t \in[0, T]}$ generated by the Brownian motion $B$.

2. Next, we approximate the drift coefficient $b=\tilde{b}+\hat{b}$ by a sequence of functions (which always exists by standard approximation results)

$$
b_{n}:=\tilde{b}_{n}+\hat{b}, \quad n \geq 1
$$

such that $\left\{\tilde{b}_{n}\right\}_{n \geq 1} \subset C_{0}^{\infty}([0, T] \times \mathbb{R})$ with $\sup _{n \geq 1}\left\|\tilde{b}_{n}\right\|_{\infty} \leq C<\infty$ and further $\tilde{b}_{n} \rightarrow \tilde{b}$ in $(t, x) \in[0, T] \times \mathbb{R}$ a.e. with respect to the Lebesgue measure. By standard results on SDE's, we know that for each regular coefficient $b_{n}, n \geq 1$, there exists a unique strong solution $X^{n, x}$ to the SDE

$$
d X_{t}^{n, x}=b_{n}\left(t, X_{t}^{n, x}\right) d t+d B_{t}, \quad 0 \leq t \leq T, \quad X_{0}^{n, x}=x \in \mathbb{R} .
$$

We then show that for each $t \in[0, T]$ the sequence $X_{t}^{n, x}$ converges weakly to the conditional expectation $E\left[X_{t}^{x} \mid \mathcal{F}_{t}\right]$ in the space $L^{2}\left(\Omega ; \mathcal{F}_{t}\right)$ of square integrable, $\mathcal{F}_{t}$-measurable random variables.

3. By Proposition 2.4 we know that for each $t \in[0, T]$ the strong solutions $X_{t}^{n, x}, n \geq 1$, are Malliavin differentiable with

$$
D_{s} X_{t}^{n, x}=\exp \left\{\int_{s}^{t} b_{n}^{\prime}\left(u, X_{u}^{n, x}\right) d u\right\}, \quad 0 \leq s \leq t \leq T, \quad n \geq 1,
$$

where $b_{n}^{\prime}$ denotes the derivative of $b_{n}$ with respect to $x$. We will use representation 222 to employ the compactness criterion 2.3 to show that for every $t \in[0, T]$ the set of random variables $\left\{X_{t}^{n, x}\right\}_{n \geq 1}$ is relatively compact in $L^{2}\left(\Omega ; \mathcal{F}_{t}\right)$, which then allows to conclude that $X_{t}^{n, x}$ converges strongly in $L^{2}\left(\Omega ; \mathcal{F}_{t}\right)$ to $E\left[X_{t}^{x} \mid \mathcal{F}_{t}\right]$. Further we obtain that $E\left[X_{t}^{x} \mid \mathcal{F}_{t}\right]$ is Malliavin differentiable as a consequence of the compactness criterion.

4. In the last step we show that $E\left[X_{t}^{x} \mid \mathcal{F}_{t}\right]=X_{t}^{x}$, which implies that $X_{t}^{x}$ is $\mathcal{F}_{t}$-measurable and thus a strong solution. Moreover, we show that this solution is unique.

Notation: In the following we sometimes include the drift coefficient $b$ into the sequence $\left\{b_{n}\right\}_{n \geq 0}$ by putting $b_{0}:=\tilde{b}_{0}+\hat{b}:=\tilde{b}+\hat{b}=b$.

The next important result is an explicit representation of the Malliavin derivative of the strong solution $X_{t}^{x}, t \in[0, T]$. For smooth coefficients $b$ we can explicitly express the Malliavin derivative in terms of the derivative of $b$ 
as stated in 22). For general, not necessarily differentiable coefficients $b$, we are still able to give an explicit formula which now involves the coefficient $b$ in a local time integral:

Proposition 3.2. For $0 \leq s \leq t \leq T$, the Malliavin derivative $D_{s} X_{t}^{x}$ of the unique strong solution $X_{t}^{x}$ to equation (5) has the following explicit representation:

$$
D_{s} X_{t}^{x}=\exp \left\{-\int_{s}^{t} \int_{\mathbb{R}} b(u, y) L^{X^{x}}(d u, d y)\right\},
$$

where $L^{X^{x}}(d u, d y)$ denotes integration in space and time with respect to the local time of $X^{x}$, see Section 2.2 for definitions.

Next, we turn our attention to the study of the strong solution $X_{t}^{x}$ as a function in its initial condition $x$ for SDE's with possible irregular drift coefficients. The first result establishes Hölder continuity jointly in time and space.

Proposition 3.3. Let $X_{t}^{x}, t \in[0, T]$ be the unique strong solution to the SDE (5). Then for all $s, t \in[0, T]$ and $x, y \in K$ for any arbitrary compact subset $K \subset \mathbb{R}$ there exists a constant $C=C\left(K,\|\tilde{b}\|_{\infty},\left\|\hat{b}^{\prime}\right\|_{\infty}\right)>0$ such that

$$
E\left[\left|X_{t}^{x}-X_{s}^{y}\right|^{2}\right] \leq C\left(|t-s|+|x-y|^{2}\right)
$$

In particular, there exists a continuous version of the random field $(t, x) \mapsto X_{t}^{x}$ with Hölder continuous trajectories of order $\alpha<1 / 2$ in $t \in[0, T]$ and $\alpha<1$ in $x \in \mathbb{R}$.

If the drift coefficient $b$ is sufficiently regular, then we know by Proposition 2.4 that $X_{t}^{x}$ is even differentiable as a function in $x$. The first variation process $\frac{\partial}{\partial x} X^{x}$ is then given by $(12)$. In the following we will derive analogous results for irregular drift coefficients, where in general the first variation process will now exist in the Sobolev derivative sense. Let $U \subset \mathbb{R}$ be an open and bounded subset. The Sobolev space $W^{1,2}(U)$ is defined as the set of functions $u: \mathbb{R} \rightarrow \mathbb{R}, u \in L^{2}(U)$ such that its weak derivative belongs to $L^{2}(U)$. The Sobolev space is endowed with the norm

$$
\|u\|_{1,2}=\|u\|_{2}+\left\|u^{\prime}\right\|_{2}
$$

where $u^{\prime}$ stands for the weak derivative of $u \in W^{1,2}(U)$. We say that the solution $X_{t}^{x}, t \in[0, T]$ is Sobolev differentiable in $U$ if for all $t \in[0, T], X_{t}$ belongs to $W^{1,2}(U), P$-a.s. Observe that in general $X_{t}$ is not in $W^{1,2}(\mathbb{R})$, e.g. take $b \equiv 0$.

Theorem 3.4. Let $b:[0, T] \times \mathbb{R} \rightarrow \mathbb{R}$ be as in $\sqrt{6})$. Let $X_{t}^{x}, t \in[0, T]$ be the unique strong solution to the $S D E$ (5) and $U \subset \mathbb{R}$ an open, bounded set. Then for every $t \in[0, T]$ we have

$$
\left(x \mapsto X_{t}^{x}\right) \in L^{2}\left(\Omega, W^{1,2}(U)\right) .
$$


We remark that using analogue techniques as in [30] one could even establish that the strong solution gives rise to a flow of Sobolev diffeomorphisms. This, however, is beyond the scope of this paper.

Similarly as for the Malliavin derivative, we are able to give an explicit representation for the first variation process in the Sobolev sense that does not involve the derivative of the drift coefficient by employing local time integration.

Proposition 3.5. Let $b:[0, T] \times \mathbb{R} \rightarrow \mathbb{R}$ be as in $(6)$. Then the first variation process (in the Sobolev sense) of the strong solution $X_{t}^{x}, t \in[0, T]$ to $S D E$ (5) has the following explicit representation

$$
\frac{\partial}{\partial x} X_{t}^{x}=\exp \left\{-\int_{0}^{t} \int_{\mathbb{R}} b(u, y) L^{X^{x}}(d u, d y)\right\} \quad d t \otimes P-\text { a.s. }
$$

As a direct consequence of Proposition 3.5 together with Proposition 3.2 we obtain the following relation between the Malliavin derivative and the first variation process, which is an extension of Proposition 2.4 to irregular drift coefficients and which is a key result in deriving the desired expression for the Delta.

Corollary 3.6. Let $X_{t}^{x}, t \in[0, T]$, be the unique strong solution to (5). Then the following relationship between the spatial derivative and the Malliavin derivative of $X_{t}^{x}$ holds:

$$
\frac{\partial}{\partial x} X_{t}^{x}=D_{s} X_{t}^{x} \frac{\partial}{\partial x} X_{s}^{x} \quad P-\text { a.s. }
$$

for any $s, t \in[0, T], s \leq t$.

Remark 3.7. Note that by Lemma 2.11 the Malliavin derivative in $(23)$ and the first variation process in 24 can be expressed in various alternative ways. Firstly, we observe that by formula $(17)$ the local time integral of the regular part $\hat{b}$ in $b$ can be separated and rewritten in the form

$$
-\int_{s}^{t} \int_{\mathbb{R}} b(u, y) L^{X^{x}}(d u, d y)=\int_{s}^{t} \hat{b}^{\prime}\left(u, X_{u}^{x}\right) d u-\int_{s}^{t} \int_{\mathbb{R}} \tilde{b}(u, y) L^{X^{x}}(d u, d y) \text { a.s. }
$$

If in addition $\tilde{b}(t, \cdot)$ is locally square integrable and continuous in $t$ as a map from $[0, T]$ to $L_{l o c}^{2}(\mathbb{R})$ or even time-homogeneous, then by Lemma 2.11 also the local time integral associated to the irregular part $\tilde{b}$ can be reformulated in terms of the generalised covariation process as in $(18)$ or in terms of the generalised Itô formula as in (19), respectively. In particular, these reformulations are useful for simulation purposes.

We conclude this section by giving an extension of all the results seen so far to a class of SDE's with more general diffusion coefficients. 
Theorem 3.8. Consider the time-homogeneous SDE

$$
d X_{t}^{x}=b\left(X_{t}^{x}\right) d t+\sigma\left(X_{t}^{x}\right) d B_{t}, \quad X_{0}^{x}=x \in \mathbb{R}, \quad 0 \leq t \leq T,
$$

where the coefficients $b: \mathbb{R} \longrightarrow \mathbb{R}$ and $\sigma: \mathbb{R} \longrightarrow \mathbb{R}$ are Borel measurable. Require that there exists a twice continuously differentiable bijection $\Lambda: \mathbb{R} \rightarrow \mathbb{R}$ with derivatives $\Lambda^{\prime}$ and $\Lambda^{\prime \prime}$ such that

$$
\Lambda^{\prime}(y) \sigma(y)=1 \text { for a.e. } y \in \mathbb{R},
$$

as well as

$$
\Lambda^{-1} \text { is Lipschitz continuous. }
$$

Suppose that the function $b_{*}: \mathbb{R} \longrightarrow \mathbb{R}$ given by

$$
b_{*}(x):=\Lambda^{\prime}\left(\Lambda^{-1}(x)\right) b\left(\Lambda^{-1}(x)\right)+\frac{1}{2} \Lambda^{\prime \prime}\left(\Lambda^{-1}(x)\right) \sigma\left(\Lambda^{-1}(x)\right)^{2}
$$

satisfies the conditions of Theorem 3.1. Then there exists a Malliavin differentiable strong solution $X^{x}$ to $\sqrt{27}$ ) which is (locally) Sobolev differentiable in its initial condition.

Proof. The proof is obtained directly from Itô's formula. See [28].

\section{Existence and derivative-free representations of the Delta}

We now turn to the main objective of the paper to extend Theorem 1.1 to the case of irregular drift coefficients, i.e. to establish a derivative-free, probabilistic representation for the Delta

$$
\frac{\partial}{\partial x} E\left[\Phi\left(X_{T}^{x}\right)\right]
$$

of a European option with pay-off $\Phi\left(X_{T}^{x}\right)$ for some $\Phi: \mathbb{R} \rightarrow \mathbb{R}$, where the underlying price process under the risk-neutral pricing measure is modeled by the strong solution $X^{x}$ of SDE (5) with possibly irregular drift $b$ as in (6). For this purpose, we need to show in the first place that the the first order derivative (28) exists. We remark that in general the second order derivative does not exist, and thus PDE methods to compute the Delta are not applicable for general irregular drift coefficients.

In a second step we then generalize the result to path-dependent options that allow for a time window to pertorm integration by parts, i.e. to pathdependent pay-offs in the form

$$
\Phi\left(X_{s}^{x}: T_{1}<s<T\right)
$$

for a future time $T_{1} \in(0, T]$ and some $\Phi: C\left(\left[T_{1}, T\right]\right) \rightarrow \mathbb{R}$.

We start with a preliminary result which shows that in case of a smooth pay-off function with compact support the Delta for European options exists and is continuous. 
Lemma 4.1. Let $X_{t}^{x}, t \in[0, T]$ be the strong solution to SDE (5) and $\left\{X_{t}^{n, x}\right\}_{n \geq 1}$ the corresponding approximating strong solutions of SDE (21). Let $\Phi \in C_{0}^{\infty}(\mathbb{R})$ and $T>0$ and consider the functions

$$
u_{n}(x):=E\left[\Phi\left(X_{T}^{n, x}\right)\right] \quad \text { and } \quad u(x):=E\left[\Phi\left(X_{T}^{x}\right)\right] .
$$

Consider also the function

$$
\bar{u}(x):=E\left[\Phi^{\prime}\left(X_{T}^{x}\right) \frac{\partial}{\partial x} X_{T}^{x}\right],
$$

where $\frac{\partial}{\partial x} X^{x}$ is the first variation process of $X^{x}$ introduced in 24. Then $u_{n}(x) \stackrel{n \rightarrow \infty}{\longrightarrow} u(x)$ for all $x \in \mathbb{R}$, and $u_{n}^{\prime}(x) \stackrel{n \rightarrow \infty}{\longrightarrow} \bar{u}(x)$ uniformly on compact subsets $K \subset \mathbb{R}$, where $u_{n}^{\prime}$ denotes the derivative. Thus $u \in C^{1}(\mathbb{R})$ with $u^{\prime}=\bar{u}$.

Proof. Firstly we observe that the expression in $(30)$ is well-defined. This can be seen by using Cauchy-Schwarz inequality, the fact that $\Phi \in C_{0}^{\infty}(\mathbb{R})$, and Corollary A.7

It is readily checked that $u_{n}(x) \rightarrow u(x)$ for all $x \in \mathbb{R}$ since $\Phi$ is smooth by using the mean-value theorem and $X_{t}^{n, x} \rightarrow X_{t}^{x}$ in $L^{2}(\Omega)$ as $n \rightarrow \infty$ for every $t \in[0, T]$ (see Theorem A.4).

For the smooth coefficients $b_{n}$ we know that $u_{n} \in C^{1}(\mathbb{R}), n \geq 1$, and since $\Phi^{\prime}$ is bounded and by dominated convergence we get

$$
u_{n}^{\prime}(x)=E\left[\Phi^{\prime}\left(X_{T}^{n, x}\right) \frac{\partial}{\partial x} X_{T}^{n, x}\right] .
$$

We will show that $\sup _{x \in K}\left|F_{n}(x)\right| \stackrel{n \rightarrow \infty}{\longrightarrow} 0$ for any compact subset $K \subset \mathbb{R}$, where $F_{n}(x):=\left|u_{n}^{\prime}(x)-\bar{u}(x)\right|=\left|E\left[\Phi^{\prime}\left(X_{T}^{n, x}\right) \frac{\partial}{\partial x} X_{T}^{n, x}-\Phi^{\prime}\left(X_{T}^{x}\right) \frac{\partial}{\partial x} X_{T}^{x}\right]\right|$. Indeed, by plugging in expression (24) for the first variation process and Girsanov's theorem we get

$$
\begin{aligned}
F_{n}(x) \leq \mid E\left[\Phi^{\prime}\left(B_{T}^{x}\right) \exp \left\{-\int_{0}^{T} \int_{\mathbb{R}} b_{n}(u, y) L^{B^{x}}(d u, d y)\right\} \mathcal{E}\left(b_{n}\right)_{T}\right. \\
\left.\left.-\Phi^{\prime}\left(B_{T}^{x}\right) \exp \left\{-\int_{0}^{T} \int_{\mathbb{R}} b(u, y) L^{B^{x}}(d u, d y)\right\} \mathcal{E}(b)_{T}\right)\right] \mid \\
\leq \mid E\left[\Phi ^ { \prime } ( B _ { T } ^ { x } ) \mathcal { E } ( b ) _ { T } \left(\exp \left\{-\int_{0}^{T} \int_{\mathbb{R}} b_{n}(u, y) L^{B^{x}}(d u, d y)\right\}\right.\right. \\
\left.+\left|E\left[\Phi^{\prime}\left(B_{T}^{x}\right) \exp \left\{-\int_{0}^{T} \int_{\mathbb{R}} b_{n}(v, y) L^{B^{x}}(d v, d y)\right\}\left(\mathcal{E}\left(b_{n}\right)_{T}-\mathcal{E}(b)_{T}\right)\right]\right|\right] \\
:=I_{n}+I I_{n}
\end{aligned}
$$


Here, we will show estimates for $I I_{n}$, for $I_{n}$ the argument is analogous. Similarly as in the proof of Lemma A.3. using inequality $\left|e^{x}-e^{y}\right| \leq|x-y|\left(e^{x}+e^{y}\right)$ we get

$$
\begin{aligned}
I I_{n} \lesssim & E\left[\left|\Phi^{\prime}\left(B_{T}^{x}\right)\right|\left|U_{n}\right| \exp \left\{-\int_{0}^{T} \int_{\mathbb{R}} b_{n}(v, y) L^{B^{x}}(d v, d y)\right\} \mathcal{E}\left(b_{n}\right)_{T}\right] \\
& +E\left[\left|\Phi^{\prime}\left(B_{T}^{x}\right)\right|\left|U_{n}\right| \exp \left\{-\int_{0}^{T} \int_{\mathbb{R}} b_{n}(v, y) L^{B^{x}}(d v, d y)\right\} \mathcal{E}(b)_{T}\right] \\
= & : I I_{n}^{1}+I I_{n}^{2},
\end{aligned}
$$

where

$$
U_{n}:=\int_{0}^{T}\left(\tilde{b}_{n}\left(u, B_{u}^{x}\right)-\tilde{b}\left(u, B_{u}^{x}\right)\right) d B_{u}-\frac{1}{2} \int_{0}^{T}\left(b_{n}^{2}\left(u, B_{u}^{x}\right)-b^{2}\left(u, B_{u}^{x}\right)\right) d u .
$$

We will now show that $I I_{n}^{1} \rightarrow 0$ as $n \rightarrow \infty$ uniformly in $x$ on a compact subset $K \subset \mathbb{R}$. The convergence of $I I_{n}^{2}$ then follows in the same way. Denote $p=\frac{1+\varepsilon}{\varepsilon}$ with $\varepsilon>0$ from Lemma 2.6 and use Hölder's inequality with exponent $1+\varepsilon$ on the Doléans-Dade exponential, then employ formula (17) on $\hat{b}$ in $b_{n}=\tilde{b}_{n}+\hat{b}$ and use Cauchy-Schwarz inequality successively. As a result,

$$
\begin{aligned}
I I_{n}^{1} \lesssim E\left[\mathcal{E}\left(b_{n}\right)_{T}^{1+\varepsilon}\right]^{1 /(1+\varepsilon)} E\left[\left|\Phi^{\prime}\left(B_{T}^{x}\right)\right|\right]^{1 /(2 p)} E\left[\left|U_{n}\right|^{8 p}\right]^{1 /(8 p)} \\
\times E\left[\exp \left\{-4 p \int_{0}^{T} \int_{\mathbb{R}} \tilde{b}_{n}(v, y) L^{B^{x}}(d v, d y)\right]^{1 /(4 p)}\right\} \\
\times E\left[\exp \left\{8 p \int_{0}^{T} \hat{b}^{\prime}\left(v, B_{v}^{x}\right) d v\right\}\right]^{1 /(8 p)}
\end{aligned}
$$

The first and fourth factor are bounded uniformly in $n \geq 1$ and $x \in K$ by Remark A.1 and Lemma A.2, respectively. The second and and fifth factor can be controlled since $\Phi^{\prime}$ and $\hat{b}^{\prime}$ are bounded. It remains to show that

$$
\sup _{x \in K} E\left[\left|U_{n}\right|^{8 p}\right] \stackrel{n \rightarrow \infty}{\longrightarrow} 0 .
$$

Using Minkowski's, Burkholder-Davis-Gundy's, and Hölder's inequality we can write

$$
\begin{aligned}
E\left[\left|U_{n}\right|^{8 p}\right] \lesssim & \int_{0}^{T} E\left[\left|\tilde{b}_{n}\left(u, B_{u}^{x}\right)-\tilde{b}\left(u, B_{u}^{x}\right)\right|^{8 p}\right] d u \\
& +\int_{0}^{T} E\left[\left|b_{n}^{2}\left(u, B_{u}^{x}\right)-b^{2}\left(u, B_{u}^{x}\right)\right|^{8 p}\right] d u .
\end{aligned}
$$


The integrand of the first term in $(32)$ can be rewritten as

$$
\begin{aligned}
E\left[\left|\tilde{b}_{n}\left(u, B_{u}^{x}\right)-\tilde{b}\left(u, B_{u}^{x}\right)\right|^{8 p}\right] & =\frac{1}{\sqrt{2 \pi u}} \int_{\mathbb{R}}\left|\tilde{b}_{n}(u, y)-\tilde{b}(u, y)\right|^{8 p} e^{-\frac{(y-x)^{2}}{2 u}} d y \\
& \leq \frac{1}{\sqrt{2 \pi u}} e^{\frac{x^{2}}{2 u}} \int_{\mathbb{R}}\left|\tilde{b}_{n}(u, y)-\tilde{b}(u, y)\right|^{8 p} e^{-\frac{y^{2}}{4 u}} d y,
\end{aligned}
$$

where we have used $e^{-\frac{(y-x)^{2}}{2 u}}=e^{-\frac{y^{2}}{4 u}} e^{-\frac{(y-2 x)^{2}}{4 u}} e^{\frac{x^{2}}{2 u}} \leq e^{-\frac{y^{2}}{4 u}} e^{\frac{x^{2}}{2 u}}$. Similarly, one can argue for the second term in (32). By taking the supremum over $x \in K$ for each $u \in[0, T]$, the convergence (31) follows by dominated convergence.

In sum,

$$
\sup _{x \in K} F_{n}(x) \stackrel{n \rightarrow \infty}{\longrightarrow} 0
$$

and hence $u_{n}^{\prime}(x) \stackrel{n \rightarrow \infty}{\longrightarrow} \bar{u}(x)$ uniformly on compact sets $K \subset \mathbb{R}$. Thus $u \in$ $C^{1}(\mathbb{R})$ with $u^{\prime}=\bar{u}$.

We come to our main result, which extends Theorem 1.1 to underlyings with irregular drift coefficients. In particular, when plugging in expression 24 for the first variation process, we see that the formula for the Delta in (35) below involves neither the derivative of the pay-off function $\Phi$ nor the derivative of the drift coefficient $b$. We obtain this result for pay-off functions $\Phi \in L^{q}\left(\mathbb{R} ; w_{T}\right)$, where

$$
L^{q}\left(\mathbb{R} ; w_{T}\right):=\left\{f: \mathbb{R} \rightarrow \mathbb{R} \text { measurable }: \int_{\mathbb{R}}|f(x)|^{q} w_{T}(x) d x<\infty\right\}
$$

for the weight function $w_{T}$ defined by

$$
w_{T}(x):=\exp \left(-\frac{x^{2}}{4 T}\right), \quad x \in \mathbb{R},
$$

and where the exponent $q$ depends on the drift $b$. Note that all pay-off functions of practical relevance are contained in these spaces.

Theorem 4.2. Let $X^{x}$ be the strong solution to $S D E(5)$ and $\Phi: \mathbb{R} \rightarrow \mathbb{R} a$ function in $L^{2 p}\left(\mathbb{R} ; w_{T}\right)$, where $p>1$ is the conjugate of $1+\varepsilon$ for $\varepsilon>0$ in Lemma 2.6. Then the price

$$
u(x):=E\left[\Phi\left(X_{T}^{x}\right)\right]
$$

of the associated European option is continuously differentiable in $x \in \mathbb{R}$, and its derivative, i.e. the Delta, takes the form

$$
u^{\prime}(x)=E\left[\Phi\left(X_{T}^{x}\right) \int_{0}^{T} a(s) \frac{\partial}{\partial x} X_{s}^{x} d B_{s}\right]
$$

where $\frac{\partial}{\partial x} X_{s}^{x}$ is given in 24), and $a: \mathbb{R} \rightarrow \mathbb{R}$ is any bounded, measurable function such that

$$
\int_{0}^{T} a(s) d s=1
$$


Proof. Assume first $\Phi \in C_{0}^{\infty}(\mathbb{R})$. Then by Lemma 4.1 we know that $u(x)=$ $E\left[\Phi\left(X_{T}^{x}\right)\right]$ is continuously differentiable with derivative

$$
u^{\prime}(x):=E\left[\Phi^{\prime}\left(X_{T}^{x}\right) \frac{\partial}{\partial x} X_{T}^{x}\right] .
$$

Now, by Corollary 3.6, we have for any $s \leq T$

$$
\frac{\partial}{\partial x} X_{T}^{x}=D_{s} X_{T}^{x} \frac{\partial}{\partial x} X_{s}^{x}
$$

Also recall that $D_{s} X_{T}^{x}=0$ for $s \geq T$. So, for any function $a: \mathbb{R} \rightarrow \mathbb{R}$ satisfying (36) we have

$$
\frac{\partial}{\partial x} X_{T}^{x}=\int_{0}^{T} a(s) D_{s} X_{T}^{x} \frac{\partial}{\partial x} X_{s}^{x} d s
$$

As a result,

$$
u^{\prime}(x)=E\left[\Phi^{\prime}\left(X_{T}^{x}\right) \int_{0}^{T} a(s) D_{s} X_{T}^{x} \frac{\partial}{\partial x} X_{s}^{x} d s\right]=E\left[\int_{0}^{T} a(s) D_{s} \Phi\left(X_{T}^{x}\right) \frac{\partial}{\partial x} X_{s}^{x} d s\right]
$$

where the second equality follows from the Malliavin differentiability of $\Phi\left(X_{T}^{x}\right)$ (Theorem 3.1) and the Malliavin chain rule (Lemma 2.1). Since $s \mapsto a(s) \frac{\partial}{\partial x} X_{s}^{x}$ is an adapted, square-integrable process (Corollary A.7 with $p=2$ ), the duality formula for the Malliavin derivative (Theorem 2.2) yields

$$
u^{\prime}(x)=E\left[\Phi\left(X_{T}^{x}\right) \int_{0}^{T} a(s) \frac{\partial}{\partial x} X_{s}^{x} d B_{s}\right]
$$

Now, let $\Phi \in L^{2 p}\left(\mathbb{R} ; w_{T}\right)$. By standard arguments we can approximate $\Phi$ by a sequence $\left\{\Phi_{n}\right\}_{n \geq 0} \subset C_{0}^{\infty}(\mathbb{R})$ such that $\Phi_{n} \rightarrow \Phi$ in $L^{2 p}\left(\mathbb{R} ; w_{T}\right)$ as $n \rightarrow \infty$. Define $u_{n}(x):=E\left[\Phi_{n}\left(X_{T}^{x}\right)\right]$ and $\bar{u}(x):=E\left[\Phi\left(X_{T}^{x}\right) \int_{0}^{T} a(s) \frac{\partial}{\partial x} X_{s}^{x} d B_{s}\right]$ which is well-defined by Cauchy-Schwarz inequality, Girsanov's theorem in connection with Lemma 2.6. Burkholder-Davis-Gundy's inequality in connection with Corollary A.7. Then by the first step $u_{n}^{\prime}(x)$ exists for all $n \geq 1$ and

$$
\begin{aligned}
\left|u_{n}^{\prime}(x)-\bar{u}(x)\right| & =\left|E\left[\left(\Phi_{n}\left(X_{T}^{x}\right)-\Phi\left(X_{T}^{x}\right)\right) \int_{0}^{T} a(s) \frac{\partial}{\partial x} X_{s}^{x} d B_{s}\right]\right| \\
& \leq E\left[\left|\Phi_{n}\left(X_{T}^{x}\right)-\Phi\left(X_{T}^{x}\right)\right|^{2}\right]^{1 / 2} E\left[\int_{0}^{T}\left|a(s) \frac{\partial}{\partial x} X_{s}^{x}\right|^{2} d s\right]^{1 / 2} \\
& \lesssim E\left[\left|\Phi_{n}\left(B_{T}^{x}\right)-\Phi\left(B_{T}^{x}\right)\right|^{2} \mathcal{E}\left(\int_{0}^{T} b\left(u, B_{u}^{x}\right) d B_{u}\right)\right]^{1 / 2}
\end{aligned}
$$


where we have used Cauchy-Schwarz inequality, Itô's isometry, Corollary A.7 and Girsanov's theorem in this order. By Hölder's inequality with $1+\varepsilon$ for a small enough $\varepsilon>0$ from Lemma 2.6 we then get

$$
\begin{aligned}
\left|u_{n}^{\prime}(x)-\bar{u}(x)\right| & \lesssim E\left[\left|\Phi_{n}\left(B_{T}^{x}\right)-\Phi\left(B_{T}^{x}\right)\right|^{2 \frac{1+\varepsilon}{\varepsilon}}\right]^{\frac{\varepsilon}{2(1+\varepsilon)}} E\left[\mathcal{E}(b)_{T}^{1+\varepsilon}\right]^{\frac{1}{2(1+\varepsilon)}} \\
& \lesssim E\left[\left|\Phi_{n}\left(B_{T}^{x}\right)-\Phi\left(B_{T}^{x}\right)\right|^{\frac{1+\varepsilon}{\varepsilon}}\right]^{\frac{\varepsilon}{2(1+\varepsilon)}} .
\end{aligned}
$$

For the last quantity, we get similarly as in the proof of Lemma 4.1

$$
\begin{aligned}
E\left[\left|\Phi_{n}\left(B_{T}^{x}\right)-\Phi\left(B_{T}^{x}\right)\right|^{2 \frac{1+\varepsilon}{\varepsilon}}\right] & =\frac{1}{\sqrt{2 \pi T}} \int_{\mathbb{R}}\left|\Phi_{n}(y)-\Phi(y)\right|^{2 \frac{1+\varepsilon}{\varepsilon}} e^{-\frac{(y-x)^{2}}{2 T}} d y \\
& \leq \frac{1}{\sqrt{2 \pi T}} e^{\frac{x^{2}}{2 T}} \int_{\mathbb{R}}\left|\Phi_{n}(y)-\Phi(y)\right|^{2 \frac{1+\varepsilon}{\varepsilon}} e^{-\frac{y^{2}}{4 T}} d y \\
& =\frac{1}{\sqrt{2 \pi T}} e^{\frac{x^{2}}{2 T}}\left\|\Phi_{n}-\Phi\right\|_{L^{2 p}\left(\mathbb{R} ; w_{T}\right)}^{2 p} .
\end{aligned}
$$

It follows that for any compact $K \subset \mathbb{R}$

$$
\lim _{n \rightarrow \infty} \sup _{x \in K} E\left[\left|\Phi_{n}\left(B_{T}^{x}\right)-\Phi\left(B_{T}^{x}\right)\right|^{2 \frac{1+\varepsilon}{\varepsilon}}\right]=0,
$$

and thus $u_{n}^{\prime}(x) \stackrel{n \rightarrow \infty}{\longrightarrow} \bar{u}(x)$ uniformly on compact sets. Hence $u \in C^{1}(\mathbb{R})$ with $u^{\prime}=\bar{u}$.

Next, we consider path-dependent options with pay-off in the form (29). Note that $T_{1}>0$ needs to be a future time point such that the time window $\left[0, T_{1}\right]$ allows for integration by parts as in the proof of Theorem 4.2. In that case, following the same ideas as in [16, Proposition 2.2], we can reduce the path-dependent case to the setting in Theorem 4.2. Indeed, by the Markov property of $X^{x}$, see [34, Theorem 1 and Section 4.5], we can write

$$
E\left[\Phi\left(X_{s}^{x}: T_{1}<s<T\right) \mid X_{T_{1}}^{x}\right]=\widetilde{\Phi}\left(X_{T_{1}}^{x}\right),
$$

for some Borel-measurable function $\widetilde{\Phi}: \mathbb{R} \rightarrow \mathbb{R}$. We then get:

Corollary 4.3. Let $X^{x}$ be the strong solution to $S D E(5)$, and $\Phi: C(\mathbb{R}) \rightarrow \mathbb{R}$ be such that $\widetilde{\Phi}$ in $(38)$ lies in $L^{2 p}\left(\mathbb{R} ; w_{T_{1}}\right)$ where $T_{1}>0$ and $w_{T_{1}}$ is defined in (34). Further, $p>1$ is the conjugate of $1+\varepsilon$ for $\varepsilon>0$ in Lemma 2.6. Then, the price

$$
u(x)=E\left[\Phi\left(X_{s}^{x}: T_{1}<s<T\right)\right]
$$

of the associated path-dependent option is continuously differentiable in $x \in \mathbb{R}$, and its derivative, i.e. the Delta, takes the form

$$
u^{\prime}(x)=E\left[\Phi\left(X_{s}^{x}: T_{1}<s<T\right) \int_{0}^{T_{1}} a(s) \frac{\partial}{\partial x} X_{s}^{x} d B_{s}\right]
$$

for any bounded measurable function $a: \mathbb{R} \rightarrow \mathbb{R}$ such that $\int_{0}^{T_{1}} a(s) d s=1$. 
Proof. By (38) we get

$\frac{\partial}{\partial x} E\left[\Phi\left(X_{s}^{x} ; T_{1} \leq s \leq T\right)\right]=\frac{\partial}{\partial x} E\left[\widetilde{\Phi}\left(X_{T_{1}}^{x}\right)\right]=E\left[\widetilde{\Phi}\left(X_{T_{1}}^{x}\right) \int_{0}^{T_{1}} a(s) \frac{\partial}{\partial x} X_{s}^{x} d B_{s}\right]$,

where the latter follows from Theorem 4.2 . We conclude by the tower property for the conditional expectation.

Example 1 (Discrete path-dependent options) Consider a path-dependent payoff fin the form

$$
\Phi\left(X_{T_{1}}^{x}, \ldots, X_{T_{m}}^{x}\right),
$$

for time points $0<T_{1} \leq \cdots \leq T_{m} \leq T$. By Lemma 2.6 we know that there exists a constant $C>0$ such that for all $0<\varepsilon<C$

$$
E\left[\mathcal{E}\left(\int_{0}^{T} b\left(u, B_{u}^{x}\right) d B_{u}\right)^{1+\varepsilon}\right]^{\frac{1}{1+\varepsilon}} \lesssim e^{C_{\varepsilon} x^{2}},
$$

where $C_{\varepsilon}$ are constants such that $\lim _{\varepsilon \succ 0} C_{\varepsilon}=0$. Let $0<\widetilde{\varepsilon}<C$ be such that $C_{\widetilde{\varepsilon}}<\frac{1}{8 T_{1}}$. Then if $\Phi \in L^{4 p \frac{1+\widetilde{\varepsilon}}{\varepsilon}}\left(\mathbb{R}^{m} ; \hat{w}_{T}\right)$ where $p$ is the exponent from Corollary 4.3 and

$L^{q}\left(\mathbb{R}^{m} ; \hat{w}_{T}\right):=\left\{f: \mathbb{R}^{m} \rightarrow \mathbb{R}: \int_{\mathbb{R}^{m}}|f(y)|^{q} \hat{w}_{T}(y) d y<\infty\right\}, \quad \hat{w}_{T}(y):=e^{-\frac{|y|^{2}}{8 T}}$,

the Delta exists and takes the form given in (39).

Indeed, we have to verify that $\widetilde{\Phi} \in L^{2 p}\left(\mathbb{R} ; w_{T_{1}}\right)$. Denoting by $X_{t}^{s, x}$ the solution at time $t \in[0, T]$, starting in $x \in \mathbb{R}$ at time $s<t$, we can write $\widetilde{\Phi}(x)=E\left[\Phi\left(x, X_{T_{2}}^{T_{1}, x}, \ldots, X_{T_{m}}^{T_{1}, x}\right)\right]$. Hence, Jensen's inequality, Girsanov's theorem, (40) and $|z|^{\alpha} \leq 1+|z|^{2}, \alpha \in(0,1), z \in \mathbb{R}$ yields

$$
|\widetilde{\Phi}(x)|^{2 p} w_{T_{1}}(x) \leq e^{-\frac{x^{2}}{8 T_{1}}}\left(1+E\left[\left|\Phi\left(x, B_{T_{2}-T_{1}}^{x}, \ldots, B_{T_{m}-T_{1}}^{x}\right)\right|^{2 p \frac{1+\widetilde{\varepsilon}}{\varepsilon}}\right]\right) .
$$

The first summand is integrable. For the second summand, writing the multivariate density of the Brownian vector in terms of transition densities we have

$$
\begin{aligned}
\int_{\mathbb{R}}|\widetilde{\Phi}(x)|^{2 p} w_{T_{1}}(x) d x & \lesssim 1+\int_{\mathbb{R}^{m}}|\Phi(y)|^{2 p \frac{1+\tilde{\varepsilon}}{\varepsilon}} \prod_{i=1}^{m} \exp \left(-\frac{\left(y_{i}-y_{i-1}\right)^{2}}{8 T}\right) d y \\
& \lesssim 1+\left(\int_{\mathbb{R}^{m}}|\Phi(y)|^{4 p \frac{1+\widetilde{\varepsilon}}{\varepsilon}} \exp \left(-\frac{|y|^{2}}{8 T}\right) d y\right)^{\frac{1}{2}}<\infty,
\end{aligned}
$$

where we have applied Cauchy-Schwarz inequality in the second inequality.

In [15, a Malliavin weight in (39) of the form $\int_{0}^{T_{m}} a(s) \frac{\partial}{\partial x} X_{s}^{x} d B_{s}$ where $\int_{0}^{T_{i}} a(s) d s=1$ for all $i=1, \ldots m$ has been derived for discrete path-dependent payoffs in case of smooth coefficients. We would like to remark that one could obtain the analogue result for irregular drift coefficients by generalizing the proof of Theorem 4.2 instead of resorting to the Markov property as above. 
Example 2 (Asian options) Consider an Asian pay-off starting at a future time, i.e.

$$
\Phi\left(\int_{T_{1}}^{T_{2}} X_{s}^{x} d s\right)
$$

for times $0<T_{1}<T_{2} \leq T$. Then if $\Phi \in L^{4 p \frac{1+\widetilde{\varepsilon}}{\tilde{\varepsilon}}}\left(\mathbb{R} ; \widetilde{w}_{T}\right)$ where $p$ is the exponent from Corollary 4.3 and

$$
L^{q}\left(\mathbb{R} ; \widetilde{w}_{T}\right):=\left\{f: \mathbb{R} \rightarrow \mathbb{R}: \int_{\mathbb{R}^{m}}|f(y)|^{q} \widetilde{w}_{T}(y) d y<\infty\right\}, \quad \widetilde{w}_{T}(y):=e^{-\frac{|y|^{2}}{3} T^{3}}
$$

the Delta exists and takes the form given in (39).

Indeed, similarly as in the Example 1 we get $\widetilde{\Phi}(x)=E\left[\Phi\left(\int_{T_{1}}^{T_{2}} X_{s}^{T_{1}, x} d s\right)\right] \in$ $L^{2 p}\left(\mathbb{R} ; w_{T_{1}}\right)$ from the inequality

$$
|\widetilde{\Phi}(x)|^{2 p} w_{T_{1}}(x) \leq e^{-\frac{x^{2}}{8 T_{1}}}\left(1+E\left[\left|\Phi\left(\int_{T_{1}}^{T_{2}} B_{s-T_{1}}^{x} d s\right)\right|^{2 p \frac{1+\tilde{\varepsilon}}{\varepsilon}}\right]\right),
$$

using the fact that $\int_{T_{1}}^{T_{2}} B_{s-T_{1}}^{y} d s$ is normally distributed with mean $y\left(T_{2}-T_{1}\right)$ and variance $\frac{\left(T_{2}-T_{1}\right)^{3}}{3}$.

\section{Examples and Simulations}

We complete this paper by applying the results from Section 4 to the computation of the Deltas in the regime-switching examples mentioned in the Introduction. To implement the methodology, we first employ Remark 3.7 and observe that all drift coefficients from the regime switching examples in the Introduction can be written in the form $b(t, x)=\tilde{b}(x)+\hat{b}(x)$ as in (6) such that identity 17$)$ holds for $\hat{b}(x)$ and identity 19 holds for $\tilde{b}(x)$. We thus get the following reformulation of the first variation process (24):

$$
\frac{\partial}{\partial x} X_{t}^{x}=\exp \left\{2 \tilde{\beta}\left(X_{t}^{x}\right)-2 \tilde{\beta}(x)-2 \int_{0}^{t} \tilde{b}\left(X_{s}^{x}\right) d X_{s}^{x}+\int_{0}^{t} \hat{b}^{\prime}\left(X_{u}^{x}\right) d u\right\},
$$

where $\tilde{\beta}(\cdot):=\tilde{b}(0)+\int_{0}^{\cdot} \tilde{b}(y) d y$ is a primitive of $\tilde{b}(\cdot)$. This form is convenient for simulation purposes.

5.1 Black \& Scholes model with regime-switching dividend yield

Consider an extended Black \& Scholes model where the stock pays a dividend yield that switches to a higher level when the stock value passes a certain 
threshold. That is, under the risk-neutral measure the stock price $S$ is given by

$$
S_{T}^{s_{0}}=e^{\sigma X_{T}^{\ln \left(s_{0}\right) / \sigma}}, \quad s_{0}>0
$$

where $X_{t}^{x}$ is the solution of the SDE

$$
X_{t}^{x}=x+\int_{0}^{t} b\left(X_{u}^{x}\right) d u+B_{t},
$$

with

$$
b(x):=-\lambda_{1} \mathbf{1}_{(-\infty, R)}(x)-\lambda_{2} \mathbf{1}_{[R, \infty)}(x)-\frac{\sigma}{2} .
$$

Here $\sigma, \lambda_{1}, \lambda_{2}, R \in \mathbb{R}_{+}$, and $\sigma \lambda_{1}, \sigma \lambda_{2}$ are the regime-dependent dividend yields. We can see that SDE (43) is in the required form as in (5) taking $\tilde{b}(t, x)=-\left(\lambda_{2}-\lambda_{1}\right) \mathbf{1}_{[R, \infty)}(x)$ and $\hat{b}(t, x)=-\lambda_{1}-\frac{\sigma}{2}$. Letting $\Phi:=\bar{\Phi} \circ \exp \circ \sigma$. we thus get by the chain rule

$$
\frac{\partial}{\partial s_{0}} E\left[\bar{\Phi}\left(S_{T}^{s_{0}}\right)\right]=\frac{\partial}{\partial s_{0}} E\left[\Phi\left(X_{T}^{\ln \left(s_{0}\right) / \sigma}\right)\right]=\left.\frac{1}{s_{0} \sigma} \cdot \frac{\partial}{\partial x} E\left[\Phi\left(X_{T}^{x}\right)\right]\right|_{x=\frac{\ln \left(s_{0}\right)}{\sigma}} .
$$

If $\Phi \in L_{w}^{4 p}(\mathbb{R})$ we know by Theorem 4.2 that the Delta exists, and we can compute $\frac{\partial}{\partial x} E\left[\Phi\left(X_{T}^{x}\right)\right]$ by 35 to obtain

$$
\frac{\partial}{\partial s_{0}} E\left[\bar{\Phi}\left(S_{T}^{s_{0}}\right)\right]=E\left[\bar{\Phi}\left(S_{T}^{s_{0}}\right) \int_{0}^{T} \frac{a(s)}{s_{0} \sigma} \frac{\partial}{\partial x} X_{s}^{\ln \left(s_{0}\right) / \sigma} d B_{s}\right]
$$

for any bounded measurable function $a: \mathbb{R} \rightarrow \mathbb{R}$ such that $\int_{0}^{T} a(s) d s=1$, and where $\frac{\partial}{\partial x} X_{s}^{x}$ is given by 42 with $\hat{b}^{\prime}=0$ and

$$
\tilde{\beta}(x):=\int_{0}^{x} \tilde{b}(y) d y=-\left(\lambda_{2}-\lambda_{1}\right)(x-R) \mathbf{1}_{[R, \infty)}(x) .
$$

We now consider the Delta for a call option, i.e. $\bar{\Phi}(x):=(x-K)_{+}$, and for a digital option, i.e. $\bar{\Phi}(x):=\mathbf{1}_{\{x \geq K\}}$, for some strike price $K>0$. It is easily seen that in both cases $\Phi \in L_{w}^{4 p}(\mathbb{R})$. To compute (44) by Monte Carlo, $X^{x}$ is approximated by an Euler scheme (see [33. Theorem 3.1 on the Euler scheme approximation for coefficients $b$ which are non-Lipschitz due to a set of discontinuity points with Lebesgue measure zero). As in [15] we compare the performance of (44) to the approximation of the Delta by a finite difference scheme combined with Monte Carlo:

$$
\frac{\partial}{\partial s_{0}} E\left[\bar{\Phi}\left(S_{T}^{s_{0}}\right)\right] \sim \frac{E\left[\bar{\Phi}\left(S_{T}^{s_{0}+\epsilon}\right)\right]-E\left[\bar{\Phi}\left(S_{T}^{s_{0}-\epsilon}\right)\right]}{2 \epsilon}
$$

for $\epsilon$ sufficiently small. We set the parameters $T=1, s_{0}=100, \lambda_{1}=0.5$, $\lambda_{2}=1.5, R=50, \sigma=0.1, K=94$, and the function in the Malliavin weight is chosen to be $a(t):=\frac{1}{T} \mathbb{I}_{[0, T]}(t)$. Our findings are analogue to the ones in [15]: for the continuous call option pay-off function the approximation 45 seems to be more efficient (see Figure 1), whereas for the discontinuous pay-off function 


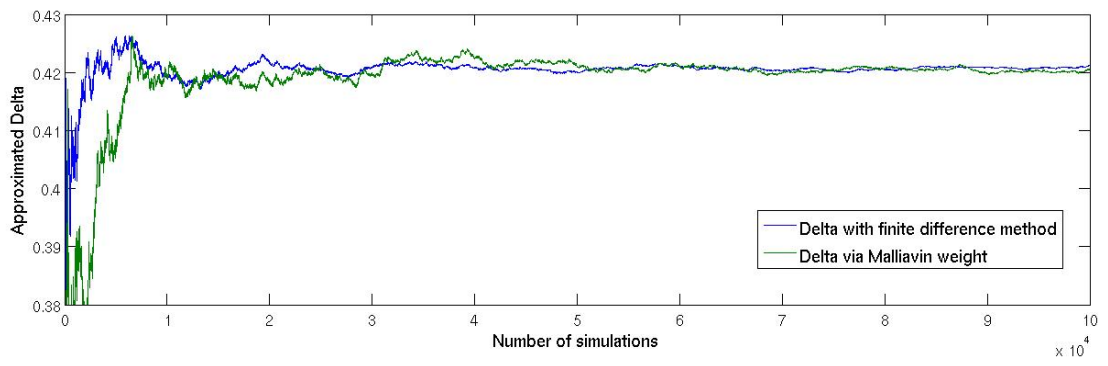

Fig. 1 Delta of a European Call Option Black \& Scholes model with regime-switching dividend yield.

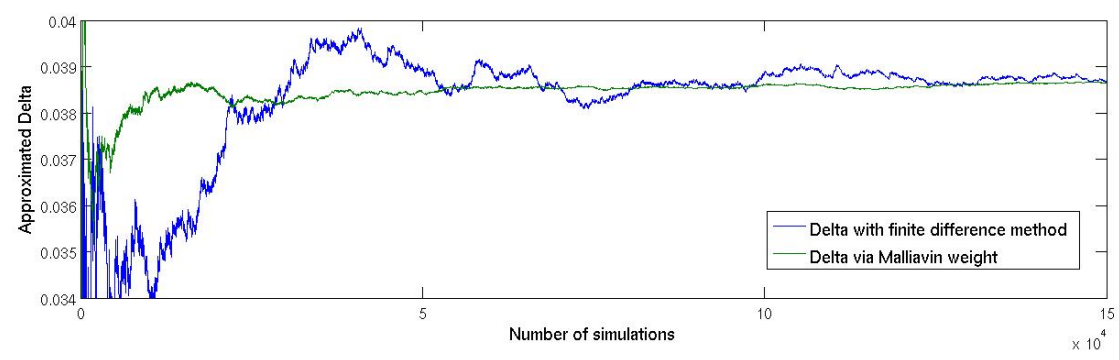

Fig. 2 Delta of a European Digital Option under the Black \& Scholes model with regimeswitching dividend yield.

of a digital option, the approximation (44) via the Malliavin weight exhibits better convergence (see Figure 2). However, this simulation study certainly does not allow for an extensive statement on the numerical properties of the involved methods.

\subsection{Electricity spot price model with regime-switching mean-reversion rate}

Typically, electricity spot prices exhibit a mean-reverting behaviour with at least two different regimes of mean-reversion: a spike regime with very strong mean-reversion on exceptionally high price levels and a base regime with moderate mean-reversion on regular price levels. These features can be captured by modelling the (de-seasonalized) electricity spot price $S$ (under a risk-neutral pricing measure) by an extended Ornstein-Uhlenbeck process with regimeswitching mean-reversion rate:

$$
S_{t}^{s_{0}}=\sigma X_{t}^{s_{0} / \sigma},
$$

where $X^{x}$ is the solution of the SDE

$$
X_{t}^{x}=x+\int_{0}^{t} b\left(X_{u}^{x}\right) d u+B_{t},
$$


with

$$
b(x):=-\left(\lambda_{1} \mathbf{1}_{(-\infty, R)}(x)+\lambda_{2} \mathbf{1}_{[R, \infty)}(x)\right) x .
$$

Here $\sigma, R, \lambda_{1}, \lambda_{2} \in \mathbb{R}_{+}, \sigma R$ denotes the spike threshold for the spot price and $\lambda_{1}, \lambda_{2}$ the regime-dependent mean-reversion rates. We observe that the $\mathrm{SDE}(47)$ is in the required form (5) with $\tilde{b}(x)=-\left(\lambda_{2}-\lambda_{1}\right) R \mathbf{1}_{[R, \infty)}(x)$ and $\hat{b}(x)=b(x)-\tilde{b}(x)$. In order to guarantee positive prices, one could alternatively model the log-price by (46), or one could introduce another regime with high mean-reversion as soon as the price falls below zero (we recall that short periods of negative electricity prices have been observed).

Since electricity is a flow commodity, derivatives on (the de-seasonalized) spot electricity are written on the average price of the delivery of $1 \mathrm{kWh}$ over a future period $\left[T_{1}, T_{2}\right]$, i.e. the underlying is of the type $\frac{1}{T_{2}-T_{1}} \int_{T_{1}}^{T_{2}} S_{t}^{s_{0}} d t$ for $T_{1}>0$. Let $\Phi$ be the pay-off function of the derivative. Then, the Delta is of Asian type and can be rewritten as:

$$
\frac{\partial}{\partial s_{0}} E\left[\Phi\left(\frac{1}{T_{2}-T_{1}} \int_{T_{1}}^{T_{2}} S_{t}^{s_{0}} d t\right)\right]=\left.\frac{1}{\sigma} \frac{\partial}{\partial x} E\left[\bar{\Phi}\left(\frac{1}{T_{2}-T_{1}} \int_{T_{1}}^{T_{2}} X_{t}^{x} d t\right)\right]\right|_{x=\frac{s_{0}}{\sigma}}
$$

with $\bar{\Phi}:=\Phi \circ \cdot \sigma$. Thus, if $\bar{\Phi}$ fulfills the integrability conditions in Example 2 the Delta exists and can be represented by formula (39). Note that in this example the first variation process $\frac{\partial}{\partial x} X_{s}^{x}$ is given by with

$$
\tilde{\beta}(x):=\int_{0}^{x} \tilde{b}(y) d y=-\left(\lambda_{2}-\lambda_{1}\right) R(x-R) \mathbf{1}_{[R, \infty)}(x)
$$

and

$$
\int_{0}^{t} \hat{b}^{\prime}\left(u, X_{u}^{x}\right) d u=-\lambda_{1} \int_{0}^{t} \mathbf{1}_{(-\infty, R)}\left(X_{u}^{x}\right) d u-\lambda_{2} \int_{0}^{t} \mathbf{1}_{[R, \infty)}\left(X_{u}^{x}\right) d u .
$$

We compare the performance of the formula (39) for the Asian Delta with a finite difference approximation analogous to 45 when $\Phi$ is a call option pay-off and a digital option pay-off, respectively. Obviously, in both cases the pay-off $\bar{\Phi}$ fulfils the assumptions in Example 2. The parameters are chosen to be $T_{1}=0.4, T_{2}=1, s_{0}=0, \lambda_{1}=0.2, \lambda_{2}=0.4, \sigma R=3, \sigma=2, K=0$, and the function $a(t)$ in the Malliavin weight is set $a(t):=\frac{1}{T_{1}} \mathbb{I}_{\left[0, T_{1}\right]}(t)$. As in Subsection 5.1, also for these Asian type options our simulations provide a more efficient convergence for the finite difference method in case of the continuous call option pay-off, see Figure 3. whereas for the discontinuous digital pay-off, the approximation through the Malliavin weight provides better convergence, see Figure 4. Again we note that this small simulation study certainly does not allow for an extensive statement on the numerical properties of the methods involved. 


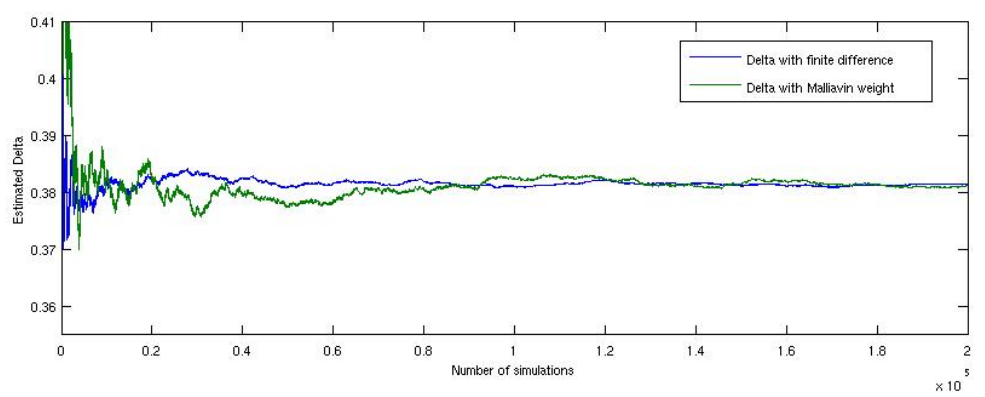

Fig. 3 Delta of an Asian Call Option on Electricity spot with regime-switching meanreversion rate.

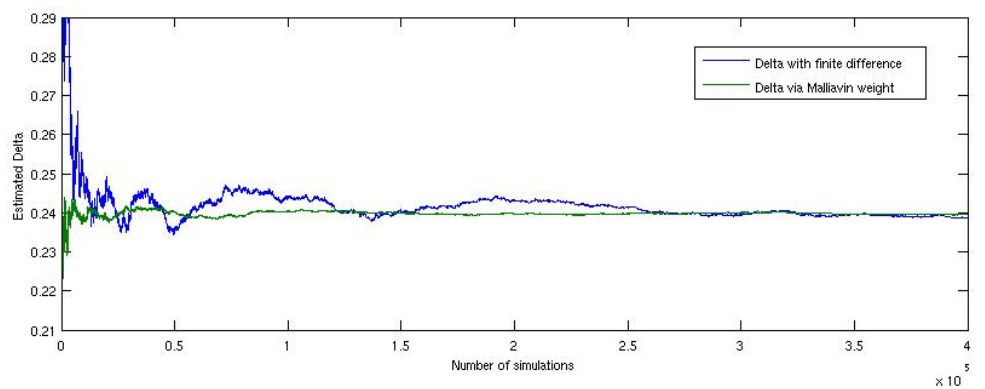

Fig. 4 Delta of an Asian Digital Option on Electricity spot with regime-switching meanreversion rate.

\section{Conclusion}

In this article we have generalised the application of Malliavin calculus to the derivation of probabilistic representations of Deltas of options with possibly discontinuous pay-offs presented in [15] to underlying diffusions with irregular drift coefficients. More precisely, while in [15] the diffusion coefficients are required to be continuously differentiable with bounded Lipschitz derivatives, we allow for drift coefficients that can be decomposed into a merely measurable and bounded and a Lipschitz continuous part.

In a first step we have established the existence of unique strong solutions of SDE's with such irregular drift coefficients that in addition are Malliavin differentiable and weakly differentiable in their initial conditions. Further, we were able to give explicit expressions of both the Malliavin and the weak derivative without employing the derivative of the drift coefficient by introducing integration in space and time with respect to local time.

In a second step, these preliminary results were used to derive the existence and probabilistic representation of Deltas of European and also pathdependent options with both possibly irregular pay-off and irregular drift coefficient. These representations neither involve the derivative of the pay-off 
function nor the derivative of the drift coefficient, whence the title Computing Deltas without Derivatives of this article.

We have finalised the article with the numerical computation of the Deltas in some examples where the drift coefficient exhibits discontinuities due to some regime-switching behaviour. To this purpose we have reformulated the local time integral in the first variation process in terms of more simulation friendly Itô integrals using a generalised Itô formula. Analogously to the results in [15], our small simulation study proposes that the Malliavin method develops its strength in comparison to a finite difference scheme in particular for discontinuous pay-offs (digital options).

\section{A Proofs of results in Section 3}

In this appendix we recollect the proofs of the results in Section 3

\section{A.1 Proof of Theorem 3.1}

We start by giving two estimates that will be progressively used throughout some proofs in the sequel.

Remark A.1. From Lemma 2.6 it follows immediately that if the approximating functions $b_{n}, n \geq 1$ are as in $(20)$ then for any compact subset $K \subset \mathbb{R}$, one can find an $\varepsilon>0$ such that

$$
\sup _{x \in K} \sup _{n \geq 0} E\left[\mathcal{E}\left(\int_{0}^{T} b_{n}\left(u, B_{u}^{x}\right) d B_{u}\right)^{1+\varepsilon}\right]<\infty
$$

where we recall that $b_{0}:=b$.

Lemma A.2. Let $f:[0, T] \times \mathbb{R} \rightarrow \mathbb{R}$ be a bounded measurable function. Then for every $t \in[0, T], \lambda \in \mathbb{R}$ and compact subset $K \subset \mathbb{R}$ we have

$$
\sup _{x \in K} E\left[\exp \left\{\lambda \int_{0}^{t} \int_{\mathbb{R}} f(s, y) L^{B^{x}}(d s, d y)\right\}\right]<\infty
$$

where $L^{B^{x}}(d s, d y)$ denotes integration with respect to local-time of the Brownian motion $B_{t}^{x}$ in both time and space, see Section 2 or [12] for more information on local-time integration. 
Proof. By virtue of decomposition (16) from Section 2 and Cauchy-Schwarz inequality twice we have

$$
\begin{aligned}
E\left[\operatorname { e x p } \left\{\lambda \int_{0}^{t} \int_{\mathbb{R}} f(s, y)\right.\right. & \left.\left.L^{B^{x}}(d s, d y)\right\}\right] \\
\leq & E\left[\exp \left\{2 \lambda \int_{0}^{t} f\left(s, B_{s}^{x}\right) d B_{s}\right\}\right]^{1 / 2} \\
& \times E\left[\exp \left\{4 \lambda \int_{T-t}^{T} f\left(T-s, B_{T-s}^{x}\right) d W_{s}\right\}\right]^{1 / 4} \\
& \times E\left[\exp \left\{-4 \lambda \int_{T-t}^{T} f\left(T-s, B_{T-s}^{x}\right) \frac{B_{T-s}}{T-s} d s\right\}\right]^{1 / 4} \\
= & I \cdot I I \cdot I I I
\end{aligned}
$$

where $W_{t}:=\int_{0}^{t} \frac{B_{T-s}}{T-s} d s+B_{T-t}-B_{T}$ was a Brownian motion. Since $f$ is bounded, factors $I$ and $I I$ are bounded uniformly in $x$ by Bernstein's inequality for exponential martingales. Finally, the boundedness of $I I I$ follows from

$$
E\left[\exp \left\{k \int_{0}^{T} \frac{\left|B_{T-s}\right|}{T-s} d s\right\}\right]<\infty
$$

for any $k \in \mathbb{R}$. To see $(52)$, using Identity 16$)$ with $f(s, y)=\operatorname{sgn}(y), x=0$ and $t=T$ in connection with Tanaka's formula we find that

$$
\int_{0}^{T} \frac{\left|\hat{B}_{s}\right|}{T-s} d s=2\left|B_{T}\right|-\int_{0}^{T} \operatorname{sgn}\left(B_{s}\right) d B_{s}+\int_{0}^{T} \operatorname{sgn}\left(\hat{B}_{s}\right) d W_{s} .
$$

Then using Hölder's inequality we obtain

$$
\begin{aligned}
E\left[\exp \left\{k \int_{0}^{T} \frac{\left|B_{T-s}\right|}{T-s} d s\right\}\right] & \\
\leq E\left[\exp \left\{6 k\left|B_{T}\right|\right\}\right]^{1 / 3} E & {\left[\exp \left\{-3 k \int_{0}^{T} \operatorname{sgn}\left(B_{s}\right) d B_{s}\right\}\right]^{1 / 3} } \\
\times E & {\left[\exp \left\{3 k \int_{0}^{T} \operatorname{sgn}\left(\widehat{B}_{s}\right) d W_{s}\right\}\right]^{1 / 3}, }
\end{aligned}
$$

and the result follows.

We now develop the proof of Theorem 3.1 according to the four-step scheme outlined in Section 3 . In order to construct a weak solution of (5) in the first step, let $(\Omega, \mathcal{F}, P)$ be some given probability space which carries a Brownian motion $\widetilde{B}$, and put $X_{t}^{x}:=\widetilde{B}_{t}+x, t \in[0, T]$. By Remark 2.5 
$\frac{d P}{d \tilde{P}}:=\mathcal{E}\left(\int_{0}^{T} b\left(u, X_{u}^{x}\right) d \widetilde{B}_{u}\right)$ defines an equivalent probability measure $P$ under which the process

$$
B_{t}:=X_{t}^{x}-x-\int_{0}^{t} b\left(s, X_{s}^{x}\right) d s, t \in[0, T]
$$

is a Brownian motion on $(\Omega, \mathcal{F}, P)$. Hence the pair $\left(X^{x}, B\right)$ is a weak solution of (5) on $(\Omega, \mathcal{F}, P)$. The stochastic basis that we operate on in the following is now given by the filtered probability space $\left(\Omega, \mathcal{F}, P,\left\{\mathcal{F}_{t}\right\}_{t \in[0, T]}\right)$, which carries the weak solution $\left(X^{x}, B\right)$ of $(5)$, where $\left\{\mathcal{F}_{t}\right\}_{t \in[0, T]}$ denotes the filtration generated by $B_{t}, t \in[0, T]$, augmented by the $P$-null sets.

The next lemma provides the second step of the proof.

Lemma A.3. Let $b_{n}:[0, T] \times \mathbb{R} \rightarrow \mathbb{R}$ be a sequence of functions approximating $b$ a.e. as in (20) and $X_{t}^{n, x}$ the corresponding strong solutions to (21), $n \geq 1$. Then for every $t \in[0, T]$ and function $\varphi \in L_{w}^{2 p}(\mathbb{R})$, where the space $L_{w}^{2 p}(\mathbb{R})$ is defined as in (33) with $p$ being the conjugate exponent of $1+\varepsilon, \varepsilon>0$ from Lemma 2.6, we have

$$
\varphi\left(X_{t}^{n, x}\right) \stackrel{n \rightarrow \infty}{\longrightarrow} E\left[\varphi\left(X_{t}^{x}\right) \mid \mathcal{F}_{t}\right]
$$

weakly in $L^{2}\left(\Omega ; \mathcal{F}_{t}\right)$.

Proof. First note that $\varphi\left(X_{t}^{n, x}\right), E\left[\varphi\left(X_{t}^{x}\right) \mid \mathcal{F}_{t}\right] \in L^{2}\left(\Omega ; \mathcal{F}_{t}\right), n \geq 0$. Indeed, Girsanov's theorem, Remark A.1 and the fact that $\varphi \in L_{w}^{2 p}(\mathbb{R})$ imply that for some constant $C_{\varepsilon}>0$ with $\varepsilon>0$ small enough we have

$$
\begin{aligned}
\sup _{n \geq 0} E\left[\left|\varphi\left(X_{t}^{n, x}\right)\right|^{2}\right] & \leq C_{\varepsilon} E\left[\left|\varphi\left(x+B_{t}\right)\right|^{2 \frac{1+\varepsilon}{\varepsilon}}\right]^{\frac{\varepsilon}{1+\varepsilon}} \\
& =C_{\varepsilon} \frac{1}{\sqrt{2 \pi t}} \int_{\mathbb{R}}|\varphi(x+z)|^{2 \frac{1+\varepsilon}{\varepsilon}} e^{-\frac{|z|^{2}}{2 T}} d z<\infty .
\end{aligned}
$$

To show the weak convergence it suffices to show

$$
\mathcal{W}\left(\varphi\left(X_{t}^{n, x}\right)\right)(f) \stackrel{n \rightarrow \infty}{\longrightarrow} \mathcal{W}\left(E\left[\varphi\left(X_{t}^{x}\right) \mid \mathcal{F}_{t}\right](f)\right.
$$

for every $f \in L^{2}([0, T])$. Indeed, by Girsanov's theorem we can write

$$
\begin{aligned}
\mathcal{W}\left(\varphi\left(X_{t}^{n, x}\right)\right)(f)-\mathcal{W}\left(E\left[\varphi\left(X_{t}^{x}\right) \mid \mathcal{F}_{t}\right](f)=\right. \\
=E\left[\varphi ( B _ { t } ^ { x } ) \left(\mathcal{E}\left(\int_{0}^{T}\left(b_{n}\left(u, B_{u}^{x}\right)+f(u)\right) d B_{u}\right)\right.\right. \\
\left.\left.-\mathcal{E}\left(\int_{0}^{T}\left(b\left(u, B_{u}^{x}\right)+f(u)\right) d B_{u}\right)\right)\right] .
\end{aligned}
$$


Using inequality $\left|e^{x}-e^{y}\right| \leq|x-y|\left(e^{x}+e^{y}\right), x, y \in \mathbb{R}$ we get

$$
\begin{aligned}
E\left[\left(\varphi\left(X_{t}^{n, x}\right)-E\left[\varphi\left(X_{t}^{x}\right) \mid \mathcal{F}_{t}\right]\right) \mathcal{E}\left(\int_{0}^{T} f(u) d B_{u}\right)\right] \\
\leq E\left[| \varphi ( B _ { t } ^ { x } ) | | U _ { n } | \left(\mathcal{E}\left(\int_{0}^{T}\left(b_{n}\left(u, B_{u}^{x}\right)+f(u)\right) d B_{u}\right)\right.\right. \\
\left.\left.+\mathcal{E}\left(\int_{0}^{T}\left(b\left(u, B_{u}^{x}\right)+f(u)\right) d B_{u}\right)\right)\right],
\end{aligned}
$$

where

$$
\begin{aligned}
U_{n}:=\int_{0}^{T}\left(b_{n}\left(u, B_{u}^{x}\right)\right. & \left.-b\left(u, B_{u}^{x}\right)\right) d B_{u} \\
& -\frac{1}{2} \int_{0}^{T}\left[\left(b_{n}\left(u, B_{u}^{x}\right)+f(u)\right)^{2}-\left(b\left(u, B_{u}^{x}\right)+f(u)\right)^{2}\right] d u .
\end{aligned}
$$

Hölder's inequality with exponents $p=\frac{1+\varepsilon}{\varepsilon}$ and $q=1+\varepsilon$ and then CauchySchwarz's inequality yields

$$
\begin{aligned}
& E\left[\left(\varphi\left(X_{t}^{n, x}\right)-E\left[\varphi\left(X_{t}^{x}\right) \mid \mathcal{F}_{t}\right]\right) \mathcal{E}\left(\int_{0}^{T} f(u) d B_{u}\right)\right] \leq \\
& \leq E\left[\left|\varphi\left(B_{t}^{x}\right)\right|^{2 \frac{1+\varepsilon}{\varepsilon}}\right]^{\frac{\varepsilon}{2(1+\varepsilon)}} E\left[\left|U_{n}\right|^{2 \frac{1+\varepsilon}{\varepsilon}}\right]^{\frac{\varepsilon}{2(1+\varepsilon)}} \\
& \quad \times E\left[\left(\mathcal{E}\left(\int_{0}^{T}\left(b_{n}\left(u, B_{u}^{x}\right)+f(u)\right) d B_{u}\right)\right.\right. \\
& \left.\left.\quad+\mathcal{E}\left(\int_{0}^{T}\left(b\left(u, B_{u}^{x}\right)+f(u)\right) d B_{u}\right)\right)^{1+\varepsilon}\right]^{\frac{1}{1+\varepsilon}} \\
& =: I^{1} \cdot I_{n}^{2} \cdot I_{n}^{3},
\end{aligned}
$$

where $\varepsilon>0$ is such that $I_{n}^{3}$ is bounded uniformly in $n \geq 0$ (see Remark A.1). The first factor $I^{1}$ is controlled as shown in (54). For the second factor $I_{n}^{2}$, using Minkowski's inequality, Burkholder-Davis-Gundy's inequality and Hölder's inequality we can write

$$
\begin{aligned}
\left(I_{n}^{2}\right)^{2 p} \lesssim \int_{0}^{T} E\left[\left|b_{n}\left(u, B_{u}^{x}\right)-b\left(u, B_{u}^{x}\right)\right|^{p}\right] d u & \\
& +\int_{0}^{T} E\left[\left|\left(b_{n}\left(u, B_{u}^{x}\right)+f(u)\right)^{2}-\left(b\left(u, B_{u}^{x}\right)+f(u)\right)^{2}\right|^{2 p}\right] d u
\end{aligned}
$$

and by dominated convergence we obtain $I_{n}^{2} \rightarrow 0$ as $n \rightarrow \infty$.

We now turn to the third step of our scheme to prove Theorem 3.1 
Theorem A.4. Let $b_{n}:[0, T] \times \mathbb{R} \rightarrow \mathbb{R}, n \geq 1$, be as in (20) and $X^{n, x}$ the corresponding strong solutions to [21). Then, for each $t \in[0, T]$

$$
X_{t}^{n, x} \stackrel{L^{2}\left(\Omega ; \mathcal{F}_{t}\right)}{\longrightarrow} E\left[X_{t}^{x} \mid \mathcal{F}_{t}\right]
$$

as $n \rightarrow \infty$. Moreover, the right-hand side of (55) is Malliavin differentiable.

Proof. The main step is to show relative compactness of $\left\{X_{t}^{n, x}\right\}_{n \geq 1}$ by applying Proposition 2.3. Let $t \in[0, T], 0 \leq s \leq s^{\prime} \leq t$ and a compact set $K \subset \mathbb{R}$ be given. Using the explicit representation introduced in (22), Girsanov's theorem, the mean-value theorem, Hölder's inequality with exponent $1+\varepsilon$ for a sufficiently small $\varepsilon>0$ and Cauchy-Schwarz inequality successively we obtain

$$
\begin{aligned}
& E\left[\left(D_{s} X_{t}^{n, x}-D_{s^{\prime}} X_{t}^{n, x}\right)^{2}\right]= \\
& =E\left[\exp \left\{2 \int_{s^{\prime}}^{t} b_{n}^{\prime}\left(u, B_{u}^{x}\right) d u\right\}\left(\exp \left\{\int_{s}^{s^{\prime}} b_{n}^{\prime}\left(u, B_{u}^{x}\right) d u\right\}-1\right)^{2} \mathcal{E}\left(b_{n}\right)_{T}\right] \\
& \leq E\left[\exp \left\{2 \int_{s^{\prime}}^{t} b_{n}^{\prime}\left(u, B_{u}^{x}\right) d u\right\}\left(\sup _{0 \leq \theta \leq 1} \exp \left\{\theta \int_{s}^{s^{\prime}} b_{n}^{\prime}\left(u, B_{u}^{x}\right) d u\right\}\right)^{2}\right. \\
& \left.\times\left(\int_{s}^{s^{\prime}} b_{n}^{\prime}\left(u, B_{u}^{x}\right) d u\right)^{2} \mathcal{E}\left(b_{n}\right)_{T}\right] \\
& \leq E\left[\exp \left\{2 \frac{1+\varepsilon}{\varepsilon} \int_{s^{\prime}}^{t} b_{n}^{\prime}\left(u, B_{u}^{x}\right) d u\right\} \sup _{0 \leq \theta \leq 1} \exp \left\{2 \frac{1+\varepsilon}{\varepsilon} \theta \int_{s}^{s^{\prime}} b_{n}^{\prime}\left(u, B_{u}^{x}\right) d u\right\}\right. \\
& \left.\times\left|\int_{s}^{s^{\prime}} b_{n}^{\prime}\left(u, B_{u}^{x}\right) d u\right|^{2 \frac{1+\varepsilon}{\varepsilon}}\right]^{\frac{\varepsilon}{1+\varepsilon}} E\left[\mathcal{E}\left(b_{n}\right)_{T}^{1+\varepsilon}\right]^{\frac{1}{1+\varepsilon}} \\
& \leq E\left[\exp \left\{4 \frac{1+\varepsilon}{\varepsilon} \int_{s^{\prime}}^{t}\left(\tilde{b}_{n}^{\prime}\left(u, B_{u}^{x}\right)+\hat{b}^{\prime}\left(u, B_{u}^{x}\right)\right) d u\right\}\right]^{\frac{\varepsilon}{2(1+\varepsilon)}} \\
& \times E\left[\sup _{0 \leq \theta \leq 1} \exp \left\{8 \frac{1+\varepsilon}{\varepsilon} \theta \int_{s}^{s^{\prime}}\left(\tilde{b}_{n}^{\prime}\left(u, B_{u}^{x}\right)+\hat{b}^{\prime}\left(u, B_{u}^{x}\right)\right) d u\right\}\right]^{\frac{\varepsilon}{4(1+\varepsilon)}} \\
& \times E\left[\left|\int_{s}^{s^{\prime}}\left(\tilde{b}_{n}^{\prime}\left(u, B_{u}^{x}\right)+\hat{b}^{\prime}\left(u, B_{u}^{x}\right)\right) d u\right|^{8 \frac{1+\varepsilon}{\varepsilon}}\right]^{\frac{\varepsilon}{4(1+\varepsilon)}} E\left[\mathcal{E}\left(b_{n}\right)_{T}^{1+\varepsilon}\right]^{\frac{1}{1+\varepsilon}} \\
& =: I_{n}^{1} \cdot I_{n}^{2} \cdot I_{n}^{3} \cdot I_{n}^{4} \text {. }
\end{aligned}
$$

Here, by Remark A.1, $\varepsilon>0$ is chosen such that $\sup _{x \in K} \sup _{n \geq 0} I_{n}^{4}<\infty$. For $I_{n}^{1}$ and $I_{n}^{2}$ we use Cauchy-Schwarz inequality and the fact that $\hat{b}^{\prime}$ is bounded and get

$$
I_{n}^{1} \lesssim E\left[\exp \left\{4 \frac{1+\varepsilon}{\varepsilon} \int_{s^{\prime}}^{t} \tilde{b}_{n}^{\prime}\left(u, B_{u}^{x}\right) d u\right\}\right]^{\frac{\varepsilon}{2(1+\varepsilon)}}=: I I_{n}^{1}
$$


and

$$
I_{n}^{2} \lesssim E\left[\sup _{0 \leq \theta \leq 1} \exp \left\{8 \frac{1+\varepsilon}{\varepsilon} \theta \int_{s}^{s^{\prime}} \tilde{b}_{n}^{\prime}\left(u, B_{u}^{x}\right) d u\right\}\right]^{\frac{\varepsilon}{4(1+\varepsilon)}}=: I I_{n}^{2}
$$

For $I_{n}^{3}$, Minkowski's inequality and the boundedness of $\hat{b}^{\prime}$ give

$$
\begin{aligned}
I_{n}^{3} & \leq E\left[\left|\int_{s}^{s^{\prime}} \tilde{b}_{n}^{\prime}\left(u, B_{u}^{x}\right) d u\right|^{8 \frac{1+\varepsilon}{\varepsilon}}+\left|\int_{s}^{s^{\prime}} \hat{b}^{\prime}\left(u, B_{u}^{x}\right) d u\right|^{8 \frac{1+\varepsilon}{\varepsilon}}\right]^{\frac{\varepsilon}{4(1+\varepsilon)}} \\
& \lesssim E\left[\left|\int_{s}^{s^{\prime}} \tilde{b}_{n}^{\prime}\left(u, B_{u}^{x}\right) d u\right|^{\frac{1+\varepsilon}{\varepsilon}}\right]^{\frac{\varepsilon}{4(1+\varepsilon)}}+\left\|\hat{b}^{\prime}\right\|_{\infty}^{2} T\left|s^{\prime}-s\right| \\
& =: I I_{n}^{3}+\left\|\hat{b}^{\prime}\right\|_{\infty}^{2} T\left|s^{\prime}-s\right| .
\end{aligned}
$$

To get rid of the derivatives $\tilde{b}_{n}^{\prime}$ in $I I_{n}^{1}, I I_{n}^{2}$ and $I I_{n}^{3}$, we use integration with respect to the local time of the Brownian motion, see Theorem 2.10 in the Section 2 and obtain

$$
\begin{aligned}
E\left[\left(D_{s} X_{t}^{n, x}-D_{s^{\prime}} X_{t}^{n, x}\right)^{2}\right] & \vdots E\left[\exp \left\{-4 \frac{1+\varepsilon}{\varepsilon} \int_{s^{\prime}}^{t} \int_{\mathbb{R}} \tilde{b}_{n}(u, y) L^{B^{x}}(d u, d y)\right\}\right]^{\frac{\varepsilon}{2(1+\varepsilon)}} \\
& \times E\left[\sup _{0 \leq \theta \leq 1} \exp \left\{-8 \frac{1+\varepsilon}{\varepsilon} \theta \int_{s}^{s^{\prime}} \int_{\mathbb{R}} \tilde{b}_{n}(u, x) L^{B^{x}}(d u, d y)\right\}\right]^{\frac{\varepsilon}{4(1+\varepsilon)}} \\
& \times\left(E\left[\left|\int_{s}^{s^{\prime}} \int_{\mathbb{R}} \tilde{b}_{n}(u, x) L^{B^{x}}(d u, d y)\right|^{8 \frac{1+\varepsilon}{\varepsilon}}\right]^{\frac{\varepsilon}{4(1+\varepsilon)}}+\left\|\hat{b}^{\prime}\right\|\left|s^{\prime}-s\right|\right)
\end{aligned}
$$

Observe that factors $I I_{n}^{1}$ and $I I_{n}^{2}$ can be controlled uniformly in $n \geq 1$ and $x \in K$ by virtue of Lemma A.2. Denote $p_{\varepsilon}:=4 \frac{1+\varepsilon}{\varepsilon}$. Then, for factor $I I_{n}^{3}$ we use (17) in connection with (16) and apply Minkowski's inequality, Burkholder-Davis-Gundy's inequality on the stochastic integrals and Hölder's 
inequality in order to obtain

$$
\begin{gathered}
I I_{n}^{3} \leq E\left[\mid-\int_{s}^{s^{\prime}} \tilde{b}_{n}\left(u, B_{u}^{x}\right) d B_{u}-\int_{T-s^{\prime}}^{T-s} \tilde{b}_{n}\left(T-u, \hat{B}_{u}^{x}\right) d W_{u}\right. \\
\left.\quad+\left.\int_{T-s^{\prime}}^{T-s} \tilde{b}_{n}\left(T-u, \hat{B}_{u}^{x}\right) \frac{\hat{B}_{u}}{T-u} d u\right|^{2 p_{\varepsilon}}\right]^{1 / p_{\varepsilon}} \\
\lesssim E\left[\left(\int_{s}^{s^{\prime}}\left|\tilde{b}_{n}\left(u, B_{u}^{x}\right)\right|^{2} d u\right)^{p_{\varepsilon}}\right]^{1 / p_{\varepsilon}} \\
+E\left[\left(\int_{T-s^{\prime}}^{T-s}\left|\tilde{b}_{n}\left(T-u, \hat{B}_{u}^{x}\right)\right|^{2} d u\right)^{p_{\varepsilon}}\right]^{1 / p_{\varepsilon}} \\
+E\left[\left|\int_{T-s^{\prime}}^{T-s} \tilde{b}_{n}\left(T-u, \hat{B}_{u}^{x}\right) \frac{\hat{B}_{u}}{T-u} d u\right|^{2 p_{\varepsilon}}\right]^{1 / p_{\varepsilon}} .
\end{gathered}
$$

Since $\tilde{b}_{n}$ is uniformly bounded we have for any $\alpha \in(0,1)$

$$
\begin{aligned}
I I_{n}^{3} & \leq\left\|\tilde{b}_{n}\right\|_{\infty}^{2}\left|s^{\prime}-s\right|+\left\|\tilde{b}_{n}\right\|_{\infty}^{2}\left(\int_{T-s^{\prime}}^{T-s}\left\|\frac{\hat{B}_{u}}{T-u}\right\|_{L^{4 p_{\varepsilon}(\Omega)}} d u\right)^{2} \\
& \leq\left\|\tilde{b}_{n}\right\|_{\infty}^{2}\left|s^{\prime}-s\right|+\left\|\tilde{b}_{n}\right\|_{\infty}^{2}\left|s^{\prime}-s\right|^{\alpha} .
\end{aligned}
$$

Altogether, we can find a constant $C>0$ depending on $T$ and $\varepsilon>0$ such that

$$
\sup _{x \in K} \sup _{n \geq 1} E\left[\left(D_{s^{\prime}} X_{t}^{n, x}-D_{s} X_{t}^{n, x}\right)^{2}\right] \leq C\left|s^{\prime}-s\right|^{\alpha}
$$

for $0 \leq s^{\prime} \leq s \leq t$ and $\alpha \in(0,1)$. Similarly, one also obtains

$$
\sup _{x \in K} \sup _{0 \leq s \leq t} \sup _{n \geq 1} E\left[\left(D_{s} X_{t}^{n, x}\right)^{2}\right] \leq C
$$

for a constant $C>0$.

Then (54) with $\varphi=i d,(56),(57)$ together with Proposition 2.3 imply that the set $\left\{X_{t}^{n, x}\right\}_{n \geq 1}$ is relatively compact in $L^{2}\left(\Omega ; \mathcal{F}_{t}\right)$. Since the sequence of solutions $X_{t}^{n, x}$ also converges weakly to $E\left[X_{t}^{x} \mid \mathcal{F}_{t}\right]$ due to Lemma A.3 with $\varphi=i d$, we get by uniqueness of the limit that

$$
X_{t}^{n_{k}, x} \stackrel{L^{2}\left(\Omega ; \mathcal{F}_{t}\right)}{\longrightarrow} E\left[X_{t}^{x} \mid \mathcal{F}_{t}\right]
$$

for a subsequence $n_{k}, k \geq 0$. Further, the $L^{2}\left(\Omega ; \mathcal{F}_{t}\right)$-convergence even holds for the whole sequence. Indeed, assume by contradiction, that there exists a subsequence $n_{j}, j \geq 0$, such that there is an $\varepsilon>0$ with $E\left[\left|X_{t}^{n_{j}, x}-X_{t}^{x}\right|^{2}\right]>\varepsilon$ for all $j \geq 0$. Then $\left\{b_{n_{j}}\right\}_{j \geq 0}$ is a sequence of approximating coefficients as required in 20. Thus, by the previous results there exists a subsequence 
$n_{j_{m}}, m \geq 0$, such that $X^{n_{j_{m}}, x} \rightarrow X^{x}$ in $L^{2}\left(\Omega ; \mathcal{F}_{t}\right)$, which gives rise to a contradiction.

Finally, since $\left\{D_{s} X_{t}^{n, x}\right\}_{n \geq 1}$ is bounded in the $L^{2}([0, T] \times \Omega)$-norm uniformly in $n$ because of (57), we also have that the limit $E\left[X_{t}^{x} \mid \mathcal{F}_{t}\right]$ is Malliavin differentiable, see e.g. 31, Lemma 1.2.3].

Remark A.5. Note that we have proved the estimates (56) and (57) uniformly in $x \in K$ for a compact set $K$ even though this is not needed to apply Proposition 2.3. We will, however, use this uniform bounds later on in the proofs of Lemma A.6 and Theorem 3.4

We are now ready to complete the proof of Theorem 3.1 by use of the previous steps.

Proof of Theorem 3.1. To prove that $X_{t}^{x}$ is a strong solution, it only remains to prove that $X_{t}^{x}$ is $\mathcal{F}_{t}$-measurable for every $t \in[0, T]$. Indeed, let $\varphi$ be a continuous bounded function. Then by Theorem A.4 we have for a subsequence $n_{k}, k \geq 0$, that

$$
\varphi\left(X_{t}^{n_{k}, x}\right) \stackrel{k \rightarrow \infty}{\longrightarrow} \varphi\left(E\left[X_{t}^{x} \mid \mathcal{F}_{t}\right]\right), \quad P-a . s .
$$

On the other side, by Lemma A.3 we also have

$$
\varphi\left(X_{t}^{n, x}\right) \rightarrow E\left[\varphi\left(X_{t}^{x}\right) \mid \mathcal{F}_{t}\right]
$$

weakly in $L^{2}\left(\Omega ; \mathcal{F}_{t}\right)$. By the uniqueness of the limit we get

$$
\varphi\left(E\left[X_{t}^{x} \mid \mathcal{F}_{t}\right]\right)=E\left[\varphi\left(X_{t}^{x}\right) \mid \mathcal{F}_{t}\right], \quad P-a . s .
$$

for all continuous, bounded functions $\varphi$, which implies that $X_{t}^{x}$ is $\mathcal{F}_{t}$-measurable for every $t \in[0, T]$.

To show uniqueness, assume that we have two strong solutions $X^{x}$ and $Y^{x}$ to the SDE (5). Then $\mathcal{W}\left(X_{t}^{x}\right)(h)=E\left[X_{t}^{x}(h)\right]$, for $h \in L^{2}([0, T])$ where $X_{t}^{x}(h), 0 \leq t \leq T$ satisfies the $\mathrm{SDE}$

$$
d X_{t}^{x}(h)=\left(b\left(t, X_{t}^{x}(h)\right)+h(t)\right) d t+d \widehat{B}_{t}, X_{0}^{x}(h)=x
$$

for a Brownian motion $\widehat{B}_{t}, 0 \leq t \leq T$. Analogously $\mathcal{W}\left(Y_{t}^{x}\right)(h)=E\left[Y_{t}^{x}(h)\right]$ with $Y_{t}^{x}(h), 0 \leq t \leq T$ solving (58). From the linear growth condition of the drift coefficient $b$ it follows that $X_{t}^{x}(h)$ and $Y_{t}^{x}(h), 0 \leq t \leq T$, are unique in law (see e.g. [19, Proposition 3.10]). Thus $\mathcal{W}\left(X_{t}^{x}\right)(h)=\mathcal{W}\left(Y_{t}^{x}\right)(h)$ for all $t, h$, and therefore $X^{x}$ and $Y^{x}$ are indistinguishable. 
A.2 Proof of Proposition 3.2

Proof of Proposition 3.2. By equation (22) and formula (17), we can write for regular coefficients $b_{n}$

$$
D_{s} X_{t}^{n, x}=\exp \left\{-\int_{s}^{t} \int_{\mathbb{R}} b_{n}(u, y) L^{X^{n, x}}(d u, d y)\right\} .
$$

Since $X_{t}^{n, x}, n \geq 1$ is relatively compact in $L^{2}\left(\Omega ; \mathcal{F}_{t}\right)$ and $\left\|D_{s} X_{t}^{n, x}\right\|_{L^{2}([0, T] \times \Omega)}$ is bounded uniformly in $n \geq 0$ due to the proof of Theorem A.4 we know that the sequence $D_{s} X_{t}^{n, x}, n \geq 1$ converges weakly to $D_{s} X_{t}^{x}$ in $L^{2}([0, T] \times \Omega)$, see [31. Lemma 1.2.3]. Therefore it is enough to check that our candidate is the weak limit, i.e. that

$$
\left\langle\mathcal{W}\left(\exp \left\{-\int^{t} \int_{\mathbb{R}} b_{n}(u, y) L^{X^{n, x}}(d u, d y)\right\}-\exp \left\{-\int^{t} \int_{\mathbb{R}} b(u, y) L^{X^{x}}(d u, d y)\right\}\right)(f), g\right\rangle_{L^{2}([0, T])} \rightarrow 0
$$

as $n \rightarrow \infty$ for every $f \in L^{2}([0, T])$ and $g \in C_{0}^{\infty}([0, T])$. It suffices to show that the Wiener transform goes to zero. As in Lemma A.3 we obtain by Girsanov's theorem that

$$
\begin{aligned}
& \mid E\left[\mathcal { E } ( \int _ { 0 } ^ { T } f ( u ) d B _ { u } ) \left(\exp \left\{-\int_{s}^{t} \int_{\mathbb{R}} b_{n}(u, y) L^{X^{n, x}}(d u, d y)\right\}\right.\right. \\
& \left.\left.-\exp \left\{-\int_{s}^{t} \int_{\mathbb{R}} b(u, y) L^{X^{x}}(d u, d y)\right\}\right)\right] \mid \\
& =\mid E\left[\exp \left\{-\int_{s}^{t} \int_{\mathbb{R}} b_{n}(u, y) L^{B^{x}}(d u, d y)\right\} \mathcal{E}\left(\int_{0}^{T}\left(b_{n}\left(u, B_{u}^{x}\right)+f(u)\right) d B_{u}\right)\right. \\
& \left.-\exp \left\{-\int_{s}^{t} \int_{\mathbb{R}} b(u, y) L^{B^{x}}(d u, d y)\right\} \mathcal{E}\left(\int_{0}^{T}\left(b\left(u, B_{u}^{x}\right)+f(u)\right) d B_{u}\right)\right] \mid \\
& \leq \mid E\left[\left(\exp \left\{-\int_{s}^{t} \int_{\mathbb{R}} \tilde{b}_{n}(u, y) L^{B^{x}}(d u, d y)\right\}\right.\right. \\
& \left.-\exp \left\{-\int_{s}^{t} \int_{\mathbb{R}} \tilde{b}(u, y) L^{B^{x}}(d u, d y)\right\}\right) \\
& \left.\times \exp \left\{\int_{s}^{t} \hat{b}^{\prime}\left(u, B_{u}^{x}\right) d u\right\} \mathcal{E}\left(\int_{0}^{T}\left(b\left(u, B_{u}^{x}\right)+f(u)\right) d B_{u}\right)\right] \mid \\
& +\mid E\left[\left(\mathcal{E}\left(\int_{0}^{T}\left(b_{n}\left(u, B_{u}^{x}\right)+f(u)\right) d B_{u}\right)-\mathcal{E}\left(\int_{0}^{T}\left(b\left(u, B_{u}^{x}\right)+f(u)\right) d B_{u}\right)\right)\right. \\
& \left.\times \exp \left\{-\int_{s}^{t} \int_{\mathbb{R}} \tilde{b}_{n}(u, y) L^{B^{x}}(d u, d y)\right\} \exp \left\{\int_{s}^{t} \hat{b}^{\prime}\left(u, B_{u}^{x}\right) d u\right\}\right] \mid \\
& =: I_{n}+I I_{n} \text {. }
\end{aligned}
$$


For term $I_{n}$ we define $p:=\frac{1+\varepsilon}{\varepsilon}$ for a suitable small $\varepsilon>0$ such that the stochastic exponential is bounded due to Lemma 2.6, and then apply Hölder's inequality with exponent $1+\varepsilon$ on the stochastic exponential. Then we apply Cauchy-Schwarz inequality and bound the factor with $\left\|\hat{b}^{\prime}\right\|_{\infty}$, and finally we use inequality $\left|e^{x}-1\right| \leq|x|\left(e^{x}+1\right)$. As a result we obtain

$$
\begin{aligned}
& I_{n}=\mid E\left[\exp \left\{-\int_{s}^{t} \int_{\mathbb{R}} \tilde{b}(u, y) L^{B^{x}}(d u, d y)\right\}\right. \\
& \times\left(\exp \left\{-\int_{s}^{t} \int_{\mathbb{R}}\left(\tilde{b}_{n}(u, y)-\tilde{b}(u, y)\right) L^{B^{x}}(d u, d y)\right\}-1\right) \\
& \left.\times \exp \left\{\int_{s}^{t} \hat{b}^{\prime}\left(u, B_{u}^{x}\right) d u\right\} \mathcal{E}\left(\int_{0}^{T}\left(b\left(u, B_{u}^{x}\right)+f(u)\right) d B_{u}\right)\right] \mid \\
& \lesssim E\left[\exp \left\{-2 p \int_{s}^{t} \int_{\mathbb{R}} \tilde{b}(u, y) L^{B^{x}}(d u, d y)\right\} \mid\right. \\
& \left.\times\left(\exp \left\{-\int_{s}^{t} \int_{\mathbb{R}}\left(\tilde{b}_{n}(u, y)-\tilde{b}(u, y)\right) L^{B^{x}}(d u, d y)\right\}-1\right)^{2 p} \mid\right]^{1 /(2 p)} \\
& \times E\left[\mathcal{E}\left(\int_{0}^{T}\left(b\left(u, B_{u}^{x}\right)+f(u)\right) d B_{u}\right)^{1+\varepsilon}\right]^{1 /(1+\varepsilon)} \\
& \lesssim E\left[| V _ { n } | ^ { 2 p } \left(\exp \left\{-\int_{s}^{t} \int_{\mathbb{R}} \tilde{b}_{n}(u, y) L^{B^{x}}(d u, d y)\right\}\right.\right. \\
& \left.\left.+\exp \left\{-\int_{s}^{t} \int_{\mathbb{R}} \tilde{b}(u, y) L^{B^{x}}(d u, d y)\right\}\right)^{2 p}\right]^{1 /(2 p)} \\
& \lesssim E\left[\left|V_{n}\right|^{2 p} \exp \left\{-2 p \int_{s}^{t} \int_{\mathbb{R}} \tilde{b}_{n}(u, y) L^{B^{x}}(d u, d y)\right\}\right]^{\frac{1}{2 p}} \\
& +E\left[\left|V_{n}\right|^{2 p} \exp \left\{-2 p \int_{s}^{t} \int_{\mathbb{R}} \tilde{b}(u, y) L^{B^{x}}(d u, d y)\right\}\right]^{\frac{1}{2 p}},
\end{aligned}
$$

where

$$
V_{n}:=\int_{s}^{t} \int_{\mathbb{R}}\left(\tilde{b}_{n}(u, y)-\tilde{b}(u, y)\right) L^{B^{x}}(d u, d y) .
$$


Cauchy-Schwarz inequality and Lemma A.2 give

$$
\begin{aligned}
E\left[\left|V_{n}\right|^{2 p}\right. & \left.\exp \left\{-2 p \int_{s}^{t} \int_{\mathbb{R}} \tilde{b}_{n}(u, y) L^{B^{x}}(d u, d y)\right\}\right] \leq \\
& \leq E\left[\left|V_{n}\right|^{4 p}\right]^{1 / 2} E\left[\exp \left\{-4 p \int_{s}^{t} \int_{\mathbb{R}} \tilde{b}_{n}(u, y) L^{B^{x}}(d u, d y)\right\}\right]^{1 / 2} \\
& \lesssim E\left[\left|V_{n}\right|^{4 p}\right]^{1 / 2} .
\end{aligned}
$$

Finally, using representation 16, Minkowski's inequality, and BurkholderDavis-Gundy's inequality we have

$$
\begin{aligned}
& E\left[\left|V_{n}\right|^{4 p}\right]=E\left[\mid \int_{s}^{t}\left(\tilde{b}_{n}\left(u, B_{u}^{x}\right)-\tilde{b}\left(u, B_{u}^{x}\right)\right) d B_{u}\right. \\
& +\int_{T-t}^{T-s}\left(\tilde{b}_{n}\left(T-u, \hat{B}_{u}^{x}\right)-\tilde{b}\left(T-u, \hat{B}_{u}^{x}\right)\right) d W_{u} \\
& \left.-\left.\int_{T-t}^{T-s}\left(\tilde{b}_{n}\left(T-u, \hat{B}_{u}^{x}\right)-\tilde{b}\left(T-u, \hat{B}_{u}^{x}\right)\right) \frac{\hat{B}_{u}}{T-u} d u\right|^{4 p}\right] \\
& \leq E\left[\left|\int_{s}^{t}\left(\tilde{b}_{n}\left(u, B_{u}^{x}\right)-\tilde{b}\left(u, B_{u}^{x}\right)\right) d B_{u}\right|^{4 p}\right] \\
& +E\left[\left|\int_{T-t}^{T-s}\left(\tilde{b}_{n}\left(T-u, \hat{B}_{u}^{x}\right)-\tilde{b}\left(T-u, \hat{B}_{u}^{x}\right)\right) d W_{u}\right|^{4 p}\right] \\
& +E\left[\left|\int_{T-t}^{T-s}\left(\tilde{b}_{n}\left(T-u, \hat{B}_{u}^{x}\right)-\tilde{b}\left(T-u, \hat{B}_{u}^{x}\right)\right) \frac{\hat{B}_{u}}{T-u} d u\right|^{4 p}\right] \\
& \leq E\left[\left(\int_{s}^{t}\left|\tilde{b}_{n}\left(u, B_{u}^{x}\right)-\tilde{b}\left(u, B_{u}^{x}\right)\right|^{2} d u\right)^{2 p}\right] \\
& +E\left[\left(\int_{T-t}^{T-s}\left|\tilde{b}_{n}\left(T-u, \hat{B}_{u}^{x}\right)-\tilde{b}\left(T-u, \hat{B}_{u}^{x}\right)\right|^{2} d u\right)^{2 p}\right] \\
& \left.+\left(\int_{T-t}^{T-s} \| \tilde{b}_{n}\left(T-u, \hat{B}_{u}^{x}\right)-\tilde{b}\left(T-u, \hat{B}_{u}^{x}\right)\right) \|_{L^{8 p}(\Omega)} \frac{1}{\sqrt{T-u}} d u\right)^{4 p} .
\end{aligned}
$$

By dominated convergence, all terms converge to zero as $n \rightarrow \infty$. The second term in 59 is estimated in the same way. Similarly, one can also bound $I I_{n}$.

\section{A.3 Proof of Proposition 3.3}

We first introduce the following auxiliary lemma. 
Lemma A.6. Let $b_{n}:[0, T] \times \mathbb{R} \rightarrow \mathbb{R}, n \geq 0$ be as in (20) and $X_{t}^{n, x}$ the corresponding strong solutions with drift coefficients $b_{n}$. Then, for any compact subset $K \subset \mathbb{R}$ and $p \geq 1$

$$
\sup _{n \geq 1} \sup _{x \in K} \sup _{t \in[0, T]} E\left[\left(\frac{\partial}{\partial x} X_{t}^{n, x}\right)^{p}\right] \leq C_{K, p}
$$

for a constant $C_{K, p}>0$ depending on $K$ and $p$. Here, $\frac{\partial}{\partial x} X_{t}^{n, x}$ is the first variation process of $X_{t}^{n, x}, n \geq 1$ (see Proposition 2.4).

Proof. The proof of this result relies on the proof of (57) in Theorem A.4 by observing that $\frac{\partial}{\partial x} X_{t}^{n, x}=D_{0} X_{t}^{n, x}$ by Proposition 2.4 . Following the same steps as in Theorem A.4 we see that all computations can be done for an arbitrary power $p \geq 1$. Finally, 61 is obtained from the term $I I_{n}^{1}$ in the proof of Theorem A.4.

Proof of Proposition 3.3. For any $p \geq 1$, the linear growth of $b_{n}$ implies

$$
E\left[\left|X_{t}^{n, x}\right|^{p}\right] \lesssim|x|^{p}+|t|+\int_{0}^{t} E\left[\left|X_{u}^{n, x}\right|^{p}\right] d u+|t|^{p / 2}
$$

Hence, Grönwall's inequality gives

$$
\sup _{n \geq 1} E\left[\left|X_{t}^{n, x}\right|^{p}\right] \leq C
$$

Let $0 \leq s<t \leq T$. Then

$$
\begin{aligned}
X_{t}^{n, x}-X_{s}^{n, y}= & x-y+\int_{s}^{t} b_{n}\left(u, X_{u}^{n, x}\right) d u \\
& +\int_{0}^{s}\left(b_{n}\left(u, X_{u}^{n, x}\right)-b_{n}\left(u, X_{u}^{n, y}\right)\right) d u+B_{t}-B_{s} .
\end{aligned}
$$

The linear growth of $b_{n}$ together with 62 and Itô's isometry then yield

$$
\begin{aligned}
E\left[\mid X_{t}^{n, x}\right. & \left.-\left.X_{s}^{n, y}\right|^{2}\right] \\
& \lesssim|x-y|^{2}+|t-s|+E\left[\left|\int_{0}^{s}\left(b_{n}\left(u, X_{u}^{n, x}\right)-b_{n}\left(u, X_{u}^{n, y}\right)\right) d u\right|^{2}\right] .
\end{aligned}
$$


Then we use the fact that $X_{t}^{n, s, \cdot}$ is a stochastic flow of diffeomorphisms (see e.g. 22] ), the mean value theorem and Lemma A.6 in order to obtain

$$
\begin{aligned}
E & {\left[\left|\int_{0}^{s}\left(b_{n}\left(u, X_{u}^{n, x}\right)-b_{n}\left(u, X_{u}^{n, y}\right)\right) d u\right|^{2}\right] } \\
& \left.=\left.|x-y|^{2} E\left[\mid \int_{0}^{s} \int_{0}^{1} b_{n}^{\prime}\left(u, X_{u}^{n, x+\tau(y-x)}\right) \frac{\partial}{\partial x} X_{u}^{n, x+\tau(y-x)}\right) d \tau d u\right|^{2}\right] \\
& \left.\left.\lesssim|x-y|^{2} \int_{0}^{1} E\left[\mid \int_{0}^{s} b_{n}^{\prime}\left(u, X_{u}^{n, x+\tau(y-x)}\right) \frac{\partial}{\partial x} X_{u}^{n, x+\tau(y-x)}\right) d u\right|^{2}\right] d \tau \\
& =|x-y|^{2} \int_{0}^{1} E\left[\left|\frac{\partial}{\partial x} X_{s}^{n, x+\tau(y-x)}-(1-\tau)\right|^{2}\right] d \tau \\
& \lesssim|x-y|^{2} \sup _{s \in[0, T]} E\left[\left|\frac{\partial}{\partial x} X_{s}^{n, x}\right|^{2}\right] \\
& \lesssim|x-y|^{2} .
\end{aligned}
$$

Altogether

$$
E\left[\left|X_{t}^{n, x}-X_{s}^{n, y}\right|^{2}\right] \lesssim|t-s|+|x-y|^{2}
$$

To conclude, we use the fact that $X_{t}^{n, x} \rightarrow X_{t}^{x}$ in $L^{2}(\Omega)$ as $n \rightarrow \infty$ due to Theorem A.4.

\section{A.4 Proof of Theorem 3.4}

Proof of Theorem 3.4. We note that for any smooth function with compact support $\varphi \in C_{0}^{\infty}(U, \mathbb{R})$ and $t \in[0, T]$, the sequence of random variables

$$
\left\langle X_{t}^{n}, \varphi\right\rangle:=\int_{U} X_{t}^{n, x} \varphi(x) d x
$$

converges weakly in $L^{2}(\Omega)$ to $\left\langle X_{t}, \varphi\right\rangle$ by using the Wiener transform following the same steps as in Lemma A.3.

Then for all measurable sets $A \in \mathcal{F}, \varphi \in C_{0}^{\infty}(\mathbb{R})$ and using Cauchy-Schwarz inequality we have

$$
\begin{aligned}
E\left[\mathbf { 1 } _ { A } \left\langleX_{t}^{n_{k}, x}\right.\right. & \left.\left.-X_{t}^{x}, \varphi^{\prime}\right\rangle\right] \\
& \leq\left\|\varphi^{\prime}\right\|_{L^{2}(U)} \mid U^{1 / 2}\left(\sup _{x \in \operatorname{supp}(U)} E\left[\mathbf{1}_{A}\left(X_{t}^{n_{k}, x}-X_{t}^{x}\right)^{2}\right]\right)^{1 / 2} \\
& <\infty
\end{aligned}
$$

where the last quantity is finite by Proposition 3.3 . Then by Theorem A.4 we see that

$$
\lim _{k \rightarrow \infty} E\left[\mathbf{1}_{A}\left\langle X_{t}^{n_{k}, x}-X_{t}^{x}, \varphi^{\prime}\right\rangle\right]=0 .
$$


In addition, by virtue of Lemma A.6 we have that

$$
\sup _{n \geq 1} E\left\|X_{t}^{n, x}\right\|_{W^{1,2}(U)}^{2}<\infty
$$

that is $\left\{x \mapsto X_{t}^{n, x}\right\}_{n \geq 1}$ is bounded in $L^{2}\left(\Omega, W^{1,2}(U)\right)$. As a result, the sequence $X_{t}^{n, x}$ is weakly relatively compact in $L^{2}\left(\Omega, W^{1,2}(U)\right)$, see e.g. 23, Theorem 10.44], and therefore there exists a subsequence $n_{k}, k \geq 0$ such that $X_{t}^{n_{k}, x}$ converges weakly to some element $Y_{t} \in L^{2}\left(\Omega, W^{1,2}(U)\right)$ as $k \rightarrow \infty$. Let us denote by $Y_{t}^{\prime}$ the weak derivative of $Y_{t}$.

Then

$$
\begin{aligned}
E\left[\mathbf{1}_{A}\left\langle X_{t}^{x}, \varphi^{\prime}\right\rangle\right] & =\lim _{k \rightarrow \infty} E\left[\mathbf{1}_{A}\left\langle X_{t}^{n_{k}, x}, \varphi^{\prime}\right\rangle\right] \\
& =-\lim _{k \rightarrow \infty} E\left[\mathbf{1}_{A}\left\langle\frac{\partial}{\partial x} X_{t}^{n_{k}, x}, \varphi\right\rangle\right] \\
& =-E\left[\mathbf{1}_{A}\left\langle Y_{t}^{\prime}, \varphi\right\rangle\right] .
\end{aligned}
$$

So

$$
\left\langle X_{t}, \varphi^{\prime}\right\rangle=-\left\langle Y_{t}^{\prime}, \varphi\right\rangle, \quad P-\text { a.s. }
$$

Finally, we need to show that there exists a measurable set $\Omega_{0} \subset \Omega$ with full measure such that $X_{t}$ has a weak derivative on this subset. To this end, choose a sequence $\left\{\varphi_{n}\right\}$ in $C^{\infty}(\mathbb{R})$ dense in $W^{1,2}(U)$. Choose a measurable subset $\Omega_{n}$ of $\Omega$ with full measure such that 63 holds on $\Omega_{n}$ with $\varphi$ replaced by $\varphi_{n}$. Then $\Omega_{0}:=\cap_{n \geq 1} \Omega_{n}$ satisfies the desired property.

Having established the existence of $\frac{\partial}{\partial x} X_{t}^{x}$ we can now extend Lemma A.6 to the case including $n=0$ :

Corollary A.7. Let $b:[0, T] \times \mathbb{R} \rightarrow \mathbb{R}$ as in (6) and $X_{t}^{x}$ the corresponding strong solution of (5). Then, for any compact subset $K \subset \mathbb{R}$ and $p \geq 1$

$$
\sup _{x \in K} \sup _{t \in[0, T]} E\left[\left(\frac{\partial}{\partial x} X_{t}^{x}\right)^{p}\right] \leq C_{K, p}
$$

for a constant $C_{K, p}>0$ depending on $K$ and $p$. Here, $\frac{\partial}{\partial x} X_{t}^{x}$ is the first variation process of $X_{t}^{x}$, (see Proposition 3.5).

Proof. This is a direct consequence of Lemma A.6 in connection with Fatou's lemma. 
A.5 Proof of Proposition 3.5

Proof of Proposition 3.5. By Theorem 3.4 the sequence $\left\{X_{t}^{n, x}\right\}_{n \geq 0}$ converges weakly to $X_{t}^{x}$ in $L^{2}\left(\Omega, W^{1,2}(U)\right)$. Therefore, it is enough to check that our candidate is the limit of $\frac{\partial}{\partial x} X_{t}^{n, x}$ in the weak topology of $L^{2}(U \times \Omega)$ for any open bounded $U \subset \mathbb{R}$. This can be shown following exactly the same steps as in Proposition 3.2 by integrating $I_{n}$ and $I I_{n}$ against $g(x)$ over $x \in U$. The only difference here is that we need all bounds uniformly in $x \in U$, which is obtained by employing Lemma A.2.

Acknowledgements We would like to thank Aaron Folly for support with the simulations in Section 5

\section{References}

1. T. Atsushi, Bismut-Elworthy-Li formulae for stochastic differential equations with jumps, Journal of Theoretical Probability 23 (2004), no. 2, 576-604.

2. D. Baños and T. Nilssen, Malliavin and flow regularity of SDEs. Application to the study of densities and the stochastic transport equation, Stochastics 88 (2016), no. 4, 540-566. MR 3473850

3. E. Benhamou, Optimal Malliavin weighting function for the computation of the Greeks, Math. Finance 13 (2003), no. 1, 37-53, Conference on Applications of Malliavin Calculus in Finance (Rocquencourt, 2001). MR 1968095

4. F. E. Benth, M. Groth, and O. Wallin, Derivative-free Greeks for the Barndorff-Nielsen and Shephard stochastic volatility model, Stochastics 82 (2010), no. 1-3, 291-313. MR 2677550 (2011k:60191)

5. F. E. Benth, J. Šaltytė Benth, and S. Koekebakker, Stochastic modelling of electricity and related markets, Advanced Series on Statistical Science \& Applied Probability, 11, World Scientific Publishing Co. Pte. Ltd., Hackensack, NJ, 2008. MR 2416686 (2009b:91001)

6. J-M. Bismut, Large deviations and the Malliavin calculus, Progress in Mathematics, vol. 45, Birkhäuser Boston, Inc., Boston, MA, 1984. MR 755001 (86f:58150)

7. R. Cont and D-A. Fournié, Functional Itô calculus and stochastic integral representation of martingales, Ann. Probab. 41 (2013), no. 1, 109-133. MR 3059194

8. G. Da Prato, P. Malliavin, and D. Nualart, Compact families of Wiener functionals, C. R. Acad. Sci. Paris Sér. I Math. 315 (1992), no. 12, $1287-$ 1291. MR 1194537 (94a:60085)

9. L. Delong, Pricing and hedging of variable annuities with state-dependent fees, Insurance Math. Econom. 58 (2014), 24-33. MR 3257334 
10. G. Di Nunno, B. Øksendal, and F. Proske, Malliavin calculus for Lévy processes with applications to finance, Universitext, Springer-Verlag, Berlin, 2009. MR 2460554 (2010f:60001)

11. B. Dupire, Functional Itô Calculus, Bloomberg Portfolio Research Paper No. 2009-04-FRONTIERS. (2009).

12. N. Eisenbaum, Integration with respect to local time, Potential Anal. 13 (2000), no. 4, 303-328. MR 1804175 (2002e:60085)

13. K. D. Elworthy and X.-M. Li, Formulae for the derivatives of heat semigroups, J. Funct. Anal. 125 (1994), no. 1, 252-286. MR 1297021 (95j:60087)

14. E. Fournié, J-M. Lasry, J. Lebuchoux, and P-L. Lions, Applications of Malliavin calculus to Monte-Carlo methods in finance. II, Finance Stoch. 5 (2001), no. 2, 201-236. MR 1841717 (2002e:91063)

15. E. Fournié, J-M. Lasry, J. Lebuchoux, P-L. Lions, and N. Touzi, Applications of Malliavin calculus to Monte Carlo methods in finance, Finance Stoch. 3 (1999), no. 4, 391-412. MR 1842285 (2002e:91062)

16. E. Gobet, Revisiting the Greeks for European and American options, Stochastic processes and applications to mathematical finance, World Sci. Publ., River Edge, NJ, 2004, pp. 53-71. MR 2202692

17. E. Gobet and R. Munos, Sensitivity analysis using Itô-Malliavin calculus and martingales, and application to stochastic optimal control, SIAM J. Control Optim. 43 (2005), no. 5, 1676-1713 (electronic). MR 2137498 (2005m:60142)

18. S. Jazaerli and Y. F. Saporito, Functional Itô Calculus, Path-dependence and the Computation of Greeks, arXiv:1311.3881 [q-fin.CP] (2015).

19. I. Karatzas and S. E. Shreve, Brownian motion and stochastic calculus, second ed., Graduate Texts in Mathematics, vol. 113, Springer-Verlag, New York, 1991. MR 1121940 (92h:60127)

20. C. Klüppelberg, T. Meyer-Brandis, and A. Schmidt, Electricity spot price modelling with a view towards extreme spike risk, Quant. Finance 10 (2010), no. 9, 963-974. MR 2738821 (2011j:91263)

21. A. Kohatsu-Higa and K. Yasuda, Estimating multidimensional density functions using the Malliavin-Thalmaier formula, SIAM J. Numer. Anal. 47 (2009), no. 2, 1546-1575. MR 2497340 (2010d:62084)

22. H. Kunita, Stochastic flows and stochastic differential equations, Cambridge Studies in Advanced Mathematics, vol. 24, Cambridge University Press, Cambridge, 1997, Reprint of the 1990 original. MR 1472487 (98e:60096)

23. G. Leoni, A first course in Sobolev spaces, Graduate Studies in Mathematics, vol. 105, American Mathematical Society, Providence, RI, 2009. MR 2527916 (2010m:46049)

24. P. Malliavin, Stochastic calculus of variation and hypoelliptic operators, Proceedings of the International Symposium on Stochastic Differential Equations (Res. Inst. Math. Sci., Kyoto Univ., Kyoto, 1976), Wiley, New York-Chichester-Brisbane, 1978, pp. 195-263. MR 536013 (81f:60083) 
25. __ Stochastic analysis, Grundlehren der Mathematischen Wissenschaften [Fundamental Principles of Mathematical Sciences], vol. 313, Springer-Verlag, Berlin, 1997. MR 1450093 (99b:60073)

26. O. Menoukeu-Pamen, T. Meyer-Brandis, T. Nilssen, F. Proske, and T. Zhang, A variational approach to the construction and Malliavin differentiability of strong solutions of SDE's, Math. Ann. 357 (2013), no. 2, 761-799. MR 3096525

27. T. Meyer-Brandis and F. Proske, On the existence and explicit representability of strong solutions of Lévy noise driven SDE's with irregular coefficients, Commun. Math. Sci. 4 (2006), no. 1, 129-154. MR 2204081 (2007b:60149)

28. _ Construction of strong solutions of SDE's via Malliavin calculus, J. Funct. Anal. 258 (2010), no. 11, 3922-3953. MR 2606880 (2011c:60189)

29. T. Meyer-Brandis and P. Tankov, Multi-factor jump-diffusion models of electricity prices, International Journal of Theoretical and Applied Finance 11 (2008), no. 5, 503-528.

30. S-E. A. Mohammed, T. K. Nilssen, and F. Proske, Sobolev differentiable stochastic flows for SDEs with singular coefficients: Applications to the transport equation, Ann. Probab. 43 (2015), no. 3, 1535-1576. MR 3342670

31. D. Nualart, The Malliavin calculus and related topics, second ed., Probability and its Applications (New York), Springer-Verlag, Berlin, 2006. MR 2200233 (2006j:60004)

32. D. Revuz and M. Yor, Continuous martingales and Brownian motion, third ed., Grundlehren der Mathematischen Wissenschaften [Fundamental Principles of Mathematical Sciences], vol. 293, Springer-Verlag, Berlin, 1999. MR 1725357 (2000h:60050)

33. L. Yan, The Euler scheme with irregular coefficients, Ann. Probab. 30 (2002), no. 3, 1172-1194. MR 1920104 (2003f:60115)

34. A. K. Zvonkin, A transformation of the phase space of a diffusion process that will remove the drift, Mat. Sb. (N.S.) 93(135) (1974), 129-149, 152. MR 0336813 (49 \#1586) 